\title{
Fission induced by nucleons at intermediate energies
}

\author{
S. Lo Meo $\left({ }^{1,4}\right)$, D. Mancusi $\left({ }^{2}\right)$, \\ C. Massimi $\left({ }^{3,4}\right)$, G. Vannini $\left({ }^{3,4}\right)$ and A. Ventura $\left({ }^{4}\right)$ \\ (1) ENEA, Centro Ricerche Ezio Clementel, 40129 Bologna, Italy \\ (2) CEA, Centre de Saclay, Irfu/SPhN, F91191 Gif-sur-Yvette CEDEX, France \\ (3) Dipartimento di Fisica ed Astronomia dell' Università di Bologna, Italy \\ (4) INFN, Sezione di Bologna, 40127 Bologna, Italy
}

June 17, 2021

\begin{abstract}
Monte Carlo calculations of fission of actinides and pre-actinides induced by protons and neutrons in the energy range from $100 \mathrm{MeV}$ to $1 \mathrm{GeV}$ are carried out by means of a recent version of the Liège Intranuclear Cascade Model, INCL++, coupled with two different evaporation-fission codes, GEMINI++ and ABLA07. In order to reproduce experimental fission cross sections, model parameters are usually adjusted on available $(p, f)$ cross sections and used to predict $(n, f)$ cross sections for the same isotopes.
\end{abstract}

\section{Introduction}

Fission induced by nucleons at intermediate energies, $i$. e. from pion production threshold $(\sim 150 \mathrm{MeV})$ to a few $\mathrm{GeV}$, is important from both basic and applied viewpoints. Even if fission is explained as a decay process of residual nuclei formed at the end of the fast nucleon cascade in the spallation reaction, many details are not yet clarified and deserve further experimental and theoretical work. 
Important applications of intermediate energy fission are energy production with accelerator driven systems [1], radioactive waste transmutation[2] and radiation shield design for accelerators: these applications require proton and neutron fission cross sections to be determined with high accuracy in a wide energy range.

Many experimental data have accumulated in the last sixty years: Ref. [3] gives a detailed review of the $(p, f)$ and $(n, f)$ measurements up to the beginning of the present century and Ref. 4 proposes a parametrization of $(p, f)$ cross sections based on the same experimental systematics.

Among the recent $(p, f)$ experiments a prominent role is played by Kotov et al. [5], who give the cross sections tabulated in steps of $100 \mathrm{MeV}$ in the range from $200 \mathrm{MeV}$ to $1 \mathrm{GeV}$ for several actinides very important for applications, ${ }^{232} \mathrm{Th},{ }^{233,235,238} \mathrm{U},{ }^{237} \mathrm{~Np}$ and ${ }^{239} \mathrm{Pu}$, and for two pre-actinides, ${ }^{n a t} \mathrm{~Pb}$ and ${ }^{209} \mathrm{Bi}$, forming the eutectic system acting as a spallation target and as a coolant in an accelerator driven system.

A similar role for $(n, f)$ experiments is currently being played by the n_TOF facility [6] at CERN, which can measure fission cross sections from thermal energies up to about $1 \mathrm{GeV}$, with a pulsed neutron beam produced by $20 \mathrm{GeV} / \mathrm{c}$ protons from the PS accelerator impinging on a lead spallation target. Since measuring an absolute cross section requires simultaneous determination of fission events and neutron flux, which is a very difficult task, the measurements performed up to the present time are relative to ${ }^{235} U(n, f)$ and absolute cross sections have been obtained by normalizing the experimental ratios to an evaluated ${ }^{235} U(n, f)$ cross section, commonly taken from the ENDF/B-VII.1 library[7] up to $E_{n}=30 \mathrm{MeV}$ and from the JENDL/HE2007 library 8 from $30 \mathrm{MeV}$ to $1 \mathrm{GeV}$. $(n, f)$ cross sections up to $1 \mathrm{GeV}$ have already been published for ${ }^{234} U$ and ${ }^{237} N p[9]$, as well as for ${ }^{n a t} P b$ and ${ }^{209} \mathrm{Bi}\left[10\right.$. Preliminary data have been obtained for ${ }^{232} \mathrm{Th}$ and ${ }^{233,238} \mathrm{U}$.

It is experimentally known[3] that at the lower extremum of the intermediate energy range $(\sim 100-150 \mathrm{MeV})$ the $(n, f)$ cross section is systematically lower than the $(p, f)$ cross section for a given target nucleus, the effect being larger for pre-actinides than for actinides, but the difference tends to decrease with increasing incident energy, so that at $1 \mathrm{GeV}$ the behaviour of protons and neutrons is expected to be quite similar and the corresponding fission cross sections of the same order.

Main purpose of the present work is to check whether available $(p, f)$ and $(n, f)$ data for a given target nucleus can be reproduced with satisfactory accuracy using the same set of model parameters, or, at least, with very 
close values; in the affirmative, where only $(p, f)$ data exist in the energy range of interest, it is reasonable to use them to predict $(n, f)$ data for the same target nucleus, or viceversa.

Our work is similar in spirit to Ref.[11], where use was made of the Los Alamos codes CEM2k+GEM2 (cascade-exciton model plus generalized evaporation model) and LAQGSM+GEM2 (quark-gluon string model plus generalized evaporation model) in order to reproduce $(p, f)$ cross sections for preactinides and actinides, from ${ }^{165} \mathrm{Ho}$ to ${ }^{239} \mathrm{Pu}$, taken mainly from Prokofiev's systematics [4], extending the calculations to a large energy range, from tens of $\mathrm{MeV}$ to $5 \mathrm{GeV}$. With CEM2k+GEM2, good fits were obtained by adjusting only two parameters, the ratio, $a_{f} / a_{n}$, of level density parameters in the fission and evaporation channels for fissioning nuclei with atomic number $70 \leq Z_{f} \leq 88$ and a constant, $C\left(Z_{f}\right)$, in a semi-empirical parametrization of the ratio, $\Gamma_{n} / \Gamma_{f}$, of neutron and fission width for $Z_{f} \geq 89$. Using the same parameters, $(n, f),(\pi, f)$ and $(\gamma, f)$ cross sections were calculated for the same nuclei and reasonable agreement with experimental data was obtained in several cases. With LAQGSM+GEM2, the set of parameters fitted from $(p, f)$ reactions made it possible to reproduce fission fragment production and spallation, fragmentation and evaporation products in heavy-ion induced reactions measured at GSI-Darmstadt in inverse kinematics 12]. Finally, the latest versions of the above mentioned codes, introduced as event generators in Los Alamos transport codes, such as MCNP6, where carefully tested not only in the calculations of fission-fragment yields and of particles emitted from them, as is usually done with transport codes, but also in the calculations of fission cross sections [13.

\section{The Models}

With the models used in the present work, fission induced by nucleons in the energy range of interest can be seen as a two-stage process: a fast cascade stage, initiated by the high energy projectile, and representable as a succession of two-body collisions, with emission of fast nucleons, light clusters, pions, etc, leaving an excited remnant, and the slow decay stage, where the remnant decays by evaporation, fission, or other mechanisms. In our system of codes, the intranuclear cascade is described by a recent $\mathrm{C}++$ version of the Liège Intranuclear Cascade Model, INCL++[14], the evaporation-fission model by a $\mathrm{C}++$ version of GEMINI, GEMINI++[19] [20], or a Fortran ver- 
sion of ABLA07[21].

It is to be pointed out, however, that the description of intermediate energy fission as a two- stage process is not a general rule in commonly used models and codes: many of them treat fission as a three-stage process, with an intermediate pre-equilibrium stage between fast cascade and evaporationfission. Such an intermediate stage was originally suggested at JINR Dubna and resulted in the original version of the cascade-exciton model[22, already mentioned in the introduction, which finally evolved in the version 23 currently used in the Los Alamos transport codes MCNPX, MCNP6 and MARS15. The three-stage description is present in the event generators of many other well-known transport codes of general use.

Coming back to the codes used in the present work, INCL++ 14 is a timelike intranuclear cascade model. At the beginning of the cascade stage, the incident nucleon is located with its own impact parameter on the surface of a working sphere, centered on the target nucleus with a radius $R_{\max }=R_{0}+8 a$, where $R_{0}$ and $a$ are the radius and the diffuseness of the target nucleus, respectively. Particles move along straight-line trajectories between collisions in the working sphere and are divided into participants and spectators in the usual sense. Participants that leave the working sphere are considered as ejectiles. Inside the working sphere, nucleons feel a potential that depends on energy and isospin. The depth of the potential well decreases linearly with increasing energy, from ordinary values at the Fermi level to zero at about $200 \mathrm{MeV}$. The isospin dependence is such that neutron and proton Fermi levels have the same energy.

Collisions are, of course, governed by Pauli blocking, treated in a different way in the first and in the subsequent collisions. The nucleons involved in the first collision are subject to a strict blocking: after the collision, both of them should lie outside the Fermi sphere. In subsequent collisions, the blocking is applied stochastically, with a probability given by the product of final-state blocking factors. A careful definition of the latter allows one to account for surface effects and for the depletion of the Fermi sphere during the evolution of the cascade.

An important novelty of recent versions of the code is the introduction of a coalescence model based on phase space, which permits the emission of light clusters, with mass $A \leq 8$, during the cascade stage, in keeping with experimental evidence.

Pions are produced in inelastic nucleon-nucleon collisions through the excitation and subsequent decay of $\Delta$ resonances, which sets an upper limit 
of the order of $3 \mathrm{GeV}$ to the incident nucleon energy for the mechanism of pion production to be valid. The lower energy limit is given by the requirement that the de Broglie wave length of relative motion be much smaller than the range of nuclear forces, which in turn is smaller than the average distance of neighbouring nucleons and is commonly set to $200 \mathrm{MeV}$, although the model performs reasonably well even at lower energies, as shown in fission calculations of the following section.

An important characteristic of the model is the self-consistent determination of the stopping time of the cascade, which can be simply parametrized as $t_{\text {stop }}=29.8 A_{T}^{0.16} \mathrm{fm} / \mathrm{c}$, with $A_{T}$ the mass of the target nucleus. At $t=t_{\text {stop }}$ many physical quantities, such as the excitation energy of the target nucleus and the average kinetic energy of the ejectiles, switch from a fast time evolution, dominated by intranuclear cascade, to a much slower evolution, which is taken as a signature of equilibration. Thanks to this choice of the stopping time, it is not necessary to introduce a pre-equilibrium model describing the intermediate stage between the fast cascade and the evaporation-fission decay. The effect of an explicit pre-equilibrium stage in the INCL model on nucleon-induced reactions above $200 \mathrm{MeV}$ was tested in Ref.[15] and found not really necessary. The effect might be more significant in the energy range from 100 to $200 \mathrm{MeV}$, with particular reference to energy spectra and angular distributions of emitted particles, rather than the fission cross sections considered in the present work. However, no clear evidence in this sense emerged from the latest IAEA Benchmark of Spallation Models[16], whose conclusions are presented in Ref.[17].

GEMINI++ [19] 20] is a statistical-model code which follows the decay of a compound nucleus by a series of sequential binary decays until such decays are forbidden by energy conservation or become improbable because of gamma-ray competition. Differently from most statistical-model codes, lightparticle evaporation is described by the Hauser-Feshbach formalism, which strictly conserves angular momentum, at the price of higher computational time with respect to the more common Weisskopf-Ewing formalism. An important ingredient of the decay width is the nuclear level density as a function of excitation energy $U$ and angular momentum $J$, described by a Bethe-type formula

$$
\rho\left(E^{*}, J\right) \sim(2 J+1) \exp \left[2 \sqrt{a\left(U\left(E^{*}\right), J\right) U}\right]
$$

Here, $E^{*}$ is the excitation energy and the thermal excitation energy $U$ is 
related to $E^{*}$ by the formula

$$
U=E^{*}-E_{\text {yrast }}(J)-\delta P-\delta W,
$$

where $E_{\text {yrast }}(J)$ is the yrast energy for angular momentum $J, \delta P$ and $\delta W$ are the pairing and shell correction, respectively, which depend on excitation energy. The level density parameter $a(U, J)$ includes the damping of shell correction $\delta W$ inspired by Ignatyuk 24$]$

$$
a(U)=\tilde{a}(U)\left[1-h\left(\frac{U}{\eta}+\frac{J}{J_{\eta}}\right) \frac{\delta W}{U}\right],
$$

with $h(x)=\tanh (x), \eta=19 \mathrm{MeV}$ and $J_{\eta}=50$. The effective level density parameter $\tilde{a}(U)$ is taken of the form 25$]$

$$
\tilde{a}(U)=\frac{A}{k_{\infty}-\left(k_{\infty}-k_{0}\right) \exp \left(-\frac{\gamma}{k_{\infty}-k_{0}} \frac{U}{A}\right)},
$$

with $k_{0}=7.3 \mathrm{MeV}, k_{\infty}=12 \mathrm{MeV}$ and $\gamma=0.00517 \exp (0.0345 A)$, with $A$ the mass number.

The decay width for symmetric fission, dominant at high excitation energy, is given by the well-known Bohr-Wheeler formula, while the decay width for asymmetric fission is derived from Moretto's formalism[26] [27], based on the concept of conditional fission barrier, $i$. e e a saddle point configuration with fixed asymmetry of mass and charge of the prefragments. Liquid drop barriers are calculated by means of Sierk's finite-range model[28], with shell and pairing corrections taken from Ref.[29].

An important adjustable parameter in the code is the ratio of the effective level density parameter at the saddle point, $\tilde{a}_{f}$, to the same quantity at ground-state deformation, $\tilde{a}_{n}$, with a default value of 1.036[20]. Fission transients are not considered, although it has been shown[20] that the model can accommodate a short fission delay.

ABLA07 21] is a statistical code describing the de-excitation of a nucleus in thermal equilibrium by particle evaporation, fission, or, above a prescribed excitation energy per nucleon, multifragmentation. Particle evaporation is treated in an extended Weisskopf-Ewing formalism, where a distribution of orbital angular momenta in the emission of nucleons and clusters is evaluated in semiclassical approximation, based on phase space arguments. The model also allows generalized evaporation of excited clusters, which plays a role analogous to asymmetric fission in GEMINI++. 
An essential ingredient of the decay formalism is the nuclear level density as a function of excitation energy and angular momentum, described by a constant temperature formula at low energy and by a Bethe-type formula (1) at high energy. The asymptotic level density parameter, $\tilde{a}$ (see formula (3)), is energy-independent and given, in $\mathrm{MeV}^{-1}$, by the original prescription of Ref. 24]

$$
\tilde{a}=0.073 A+0.095 B_{s} A^{2 / 3},
$$

where $B_{s}$ is the ratio of the surface of the deformed nucleus to that of a spherical nucleus with the same mass number $A$, as in the finite-range liquid drop model[28]. The level density contains a collective enhancement factor, due to nuclear rotations and vibrations, depending on excitation energy [30]. The approach to fission contains elements of dynamics: the time evolution of the fission degree of freedom is treated as a diffusion process, determined by the interaction of collective degrees of freedom with a heat bath formed by the individual nucleons and described by a Fokker-Planck equation whose solution leads to a time-dependent fission width, $\Gamma_{f}(t)$. An analytical approximation to such a solution and, consequently, to the time dependence of the fission width makes the problem computationally tractable. At low excitation energy, the code uses the standard model of a two-humped fission barrier, whose penetrability is computed in the approximation of full damping of the vibrational resonances in the intermediate well. Like in the GEMINI++ code, liquid drop barriers are computed in the frame of the finite-range model[28] and shell and pairing corrections are taken from Ref. [29].

\section{Fission Cross Sections}

As already stressed in the introduction, our main purpose is a simultaneous reproduction of $(p, f)$ and $(n, f)$ cross sections for the same target nuclei, possibly using the same, or, at least, rather close values of model parameters. In fact, it is expected that the isospin dependence of the fission cross section is already contained in the models we use.

In order to better understand how fission keeps memory of projectile isospin, it is worth recalling that the fission cross section increases with in-

creasing fissility parameter, $x \simeq \frac{1}{49} \frac{Z^{2}}{A}$, where $Z$ and $A$ are charge and mass of the fissioning nucleus. In the case of a proton induced reaction, the highest fissility parameter is obtained when the incident proton is captured by the 
target nucleus and is proportional to $\left(Z_{T}+1\right)^{2} /\left(A_{T}+1\right)$, with $Z_{T}$ and $A_{T}$ the charge and mass of the target nucleus, while in the case of capture of an incident neutron the fissility parameter is smaller, since it is proportional to $Z_{T}^{2} /\left(A_{T}+1\right)$

An analysis of charge and mass distributions of remnants undergoing fission at the end of the fast cascade stage, carried out in Ref. 31] with the cascade-exciton model code CEM95 32 for $(p, f)$ and $(n, f)$ reactions up to a projectile energy $E_{\text {proj }}=200 \mathrm{MeV}$ showed the relevance of remnants with charge $Z_{T}+1$ in enhancing the $(p, f)$ cross section with respect to the $(n, f)$ cross section, where remnants cannot have charges larger than $Z_{T}$. By considering the fissility parameter dependence of the liquid drop barriers, the authors of Ref.[31] explained also why the difference of $(p, f)$ and $(n, f)$ cross sections is much larger in pre-actinides than in actinides. It is worth recalling that a CEM95 analysis and interpretation of the differences between $(p, f)$ and $(n, f)$ cross sections for ${ }^{208} \mathrm{~Pb}$ and ${ }^{209} \mathrm{Bi}$ in the energy range from 45 to $500 \mathrm{MeV}$ had already been published in Ref. 333.

The analysis of differences of $(p, f)$ and $(n, f)$ reactions was further enriched with new experimental data in the pre-actinide region by the authors of Ref. [34], who derived also an approximate analytical dependence of the $\sigma_{p f} / \sigma_{n f}$ ratio on $Z_{T}^{2} / A_{T}$ at given projectile energy, $E_{\text {proj. }}$. For instance, at $E_{\text {proj }} \simeq 180 \mathrm{MeV}$, they obtained

$$
\frac{\sigma_{p f}}{\sigma_{n f}} \simeq \exp \left[0.26\left(36.6-\frac{Z_{T}^{2}}{A_{T}}\right)\right],
$$

valid for both actinides and pre-actinides. With increasing projectile energy up to the $\mathrm{GeV}$ region, it is expected that the differences in the behaviour of neutrons and protons become smaller, so that $(p, f)$ and $(n, f)$ cross sections tend to a common value.

In keeping with the philosophy of the authors of the INCL++ code [14] 18], no parameters have been modified in the intranuclear cascade model, already optimized by reproducing a large amount of data related to the cascade stage, such as total reaction cross sections, double-differential spectra of emitted nucleons, pions and light clusters, while two basic fission model parameters have been taken as adjustable either in GEMINI++ or ABLA07: the height of the liquid-drop fission barrier, $B_{f}$, and the asymptotic level density parameter at the saddle point, $\tilde{a}_{f}$, in ABLA07, or the ratio of the level density parameter at the saddle point to that at the ground state deformation, $\tilde{a}_{f} / \tilde{a}_{n}$, in GEMINI ++ . Both parameters affect significantly the calculation of fission cross 
sections in the whole energy range of interest to the present work, from 100 $\mathrm{MeV}$ to $1 \mathrm{GeV}$, although the change of the barrier height is more important at low incident energy and the change of the level density parameter at high energy. In principle, many sets of experimental data could be reproduced by modifying only one parameter, e.g. $\tilde{a}_{f}$, at the the cost of using rather different values of it in the neutron and proton channels, particularly for actinides, as we did in our preliminary work 35$]$.

In the following sub-section results for absolute $(p, f)$ and $(n, f)$ cross sections are presented and discussed in comparison with experimental data, while a separate sub-section will be dedicated to $(n, f)$ cross sections relative to ${ }^{235} U(n, f)$, with particular reference to the comparison with $\mathrm{n}_{-} \mathrm{TOF}$ data.

\subsection{Absolute Cross Sections}

The rationale behind our work is to adjust fission model parameters on the $(p, f)$ cross sections of Ref. [5] for ${ }^{n a t} \mathrm{~Pb},{ }^{209} \mathrm{Bi},{ }^{232} \mathrm{Th},{ }^{233,235,238} \mathrm{U}$ and ${ }^{239} \mathrm{Pu}$ and use similar parameter values in computing $(n, f)$ cross sections for the same targets. As alredy pointed out in our preliminary work [35], the GEMINI++ and ABLA07 models can produce fits of comparable accuracy for actinides, while in the lead-bismuth region ABLA07 appears to work better than GEMINI++. Therefore, we have decided to show the results of GEMINI++ calculations for actinides and those of ABLA07 calculations for lead and bismuth.

The experiment of Ref.[5], labelled as Kotov 2006 in the figures, yields 9 cross section values in the energy range from $207 \mathrm{MeV}$ to $1 \mathrm{GeV}$. We have

evaluated the quality of our fits by means of a $\chi^{2}$ test, where the $\chi^{2}$ variable is defined as

$$
\chi_{0}^{2}=\sum_{i=1}^{9}\left(\frac{\sigma_{i_{\text {calc. }}}-\sigma_{i \exp .}}{\Delta \sigma_{i e x p .}}\right)^{2},
$$

where the symbols are self-explanatory. Since we try to reproduce 9 experimental values, $\sigma_{\text {iexp. }}$, by adjusting 2 parameters, the relevant $\chi^{2}$ distribution, $f_{\nu}\left(\chi^{2}\right)$, should have $\nu=9-2$ degrees of freedom and the quality of fit is evaluated by means of the cumulative probability

$$
Q\left(\chi_{0}^{2}\right)=\int_{\chi_{0}^{2}}^{\infty} f_{7}\left(\chi^{2}\right) d \chi^{2}
$$




\subsubsection{Actinides}

Table 1 shows the values of the parameters adjusted on the experimental $(p, f)$ data of Ref. [5] for actinides in our INCL++/GEMINI++ calculations, $i$. e. the ratio of the level density parameter at the saddle point to the one at ground-state deformation, $\tilde{a}_{f} / \tilde{a}_{n}$, and the global correction of the liquid-drop fission barriers, $\Delta B_{f}$, in $\mathrm{MeV}$.

\begin{tabular}{|c|c|c|c|c|c|}
\hline Isotope & $Z^{2} / A$ & $\tilde{a}_{f} / \tilde{a}_{n}$ & $\Delta B_{f}(\mathrm{MeV})$ & $\chi_{0}^{2}$ & $Q\left(\chi_{0}^{2}\right)$ \\
\hline${ }^{232} \mathrm{Th}$ & 34.91 & 1.040 & -0.3 & 3.71 & 0.81 \\
\hline${ }^{238} \mathrm{U}$ & 35.56 & 1.038 & -0.3 & 4.50 & 0.72 \\
\hline${ }^{235} \mathrm{U}$ & 36.02 & 1.050 & -0.2 & 2.75 & 0.91 \\
\hline${ }^{233} \mathrm{U}$ & 36.33 & 1.100 & -0.5 & 4.16 & 0.76 \\
\hline${ }^{237} \mathrm{~Np}$ & 36.49 & 1.040 & 0.0 & 25.01 & 0.0007 \\
\hline${ }^{239} \mathrm{Pu}$ & 36.97 & 1.036 & +0.5 & 8.44 & 0.30 \\
\hline
\end{tabular}

Table 1: Model parameters and $\chi^{2}$ tests for proton-induced fission of actinides.

\subsection{2 ${ }^{235} U$}

Fig. 1 shows calculated $(p, f)$ and $(n, f)$ cross sections for ${ }^{235} U$ in comparison with experimental data.

As far as $(p, f)$ data are concerned, our reference values [5] and, consequently, our calculated cross section appear to be compatible with the old measurement of Ref.[36] (Steiner 1956) only below $150 \mathrm{MeV}$ and in agreement with the experimental point at $590 \mathrm{MeV}$ from Ref. 37] (Brandt 1972), but in conflict with Ref.[38] (Konshin 1965), which seems to have inspired the JENDL/HE-2007 evaluation, together with the more recent point at 1 GeV from Ref.[39] (Yurevich 2002).

Reliable $(n, f)$ data are available only below $200 \mathrm{MeV}$ and are taken from Refs. 40] (Lisowski 1991) and [41] (Nolte 2007), which are in mutual agreement; in both experiments fission events were detected simultaneously with $(n, p)$ scattering events, so that from knowledge of the differential $(n, p)$ scattering cross section it was possible to determine the neutron flux and, consequently, the absolute fission cross section. It is worth recalling that on the basis of the data of Ref. 40] it was proposed in Ref. [42] to extend to 200 

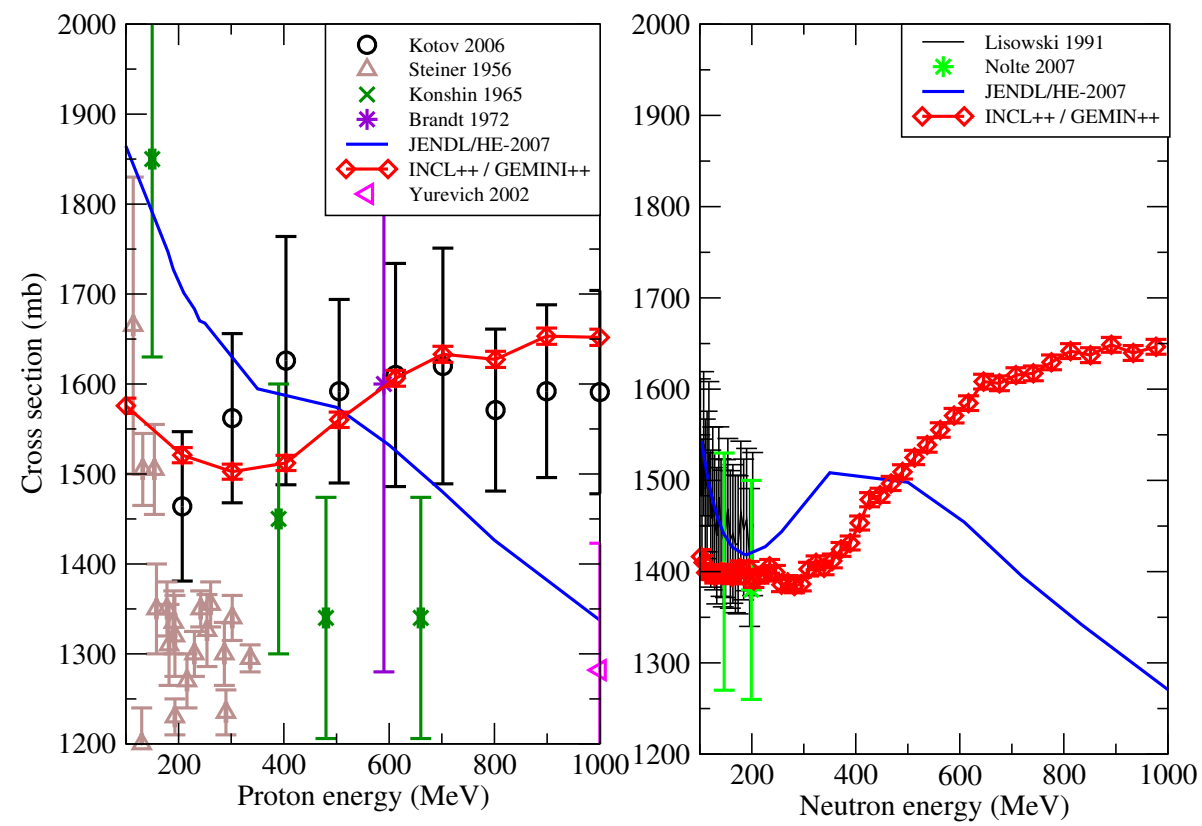

Figure 1: ${ }^{235} U$ fission cross sections. Left panel: $(p, f)$; right panel: $(n, f)$. Experimental data are discussed in the text.

MeV the energy range where the ${ }^{235} U(n, f)$ cross section can be considered as a fission standard. A previous measurement up to $750 \mathrm{MeV} 433$ yielded cross section values much lower than those of Refs. [40] [41] and has not been taken into account in this work.

The data of Refs. 40, [41] are reproduced by JENDL/HE-2007 and by us. Differently from the JENDL evaluation, however, our $(p, f)$ and $(n, f)$ cross sections show a large plateau above $500 \mathrm{MeV}$ and tend to a common value at $1 \mathrm{GeV}$. It is worth pointing out that our $(p, f)$ and $(n, f)$ calculations are done with the same model parameters, namely $\tilde{a}_{f} / \tilde{a}_{n}=1.050$ and a common reduction of all the barrier heights of remnants in both processes by an amount $\Delta B_{f}=-0.2 \mathrm{MeV}$.

\subsection{3 ${ }^{238} U$}

Similar calculations, done for ${ }^{238} U$, are shown in Fig. 2. Our $(p, f)$ cross section, adjusted on Kotov's data[5], appears to be compatible with the old data of Ref. [36] below $150 \mathrm{MeV}$ and with the recent data of Ref. [44] (Schmidt 

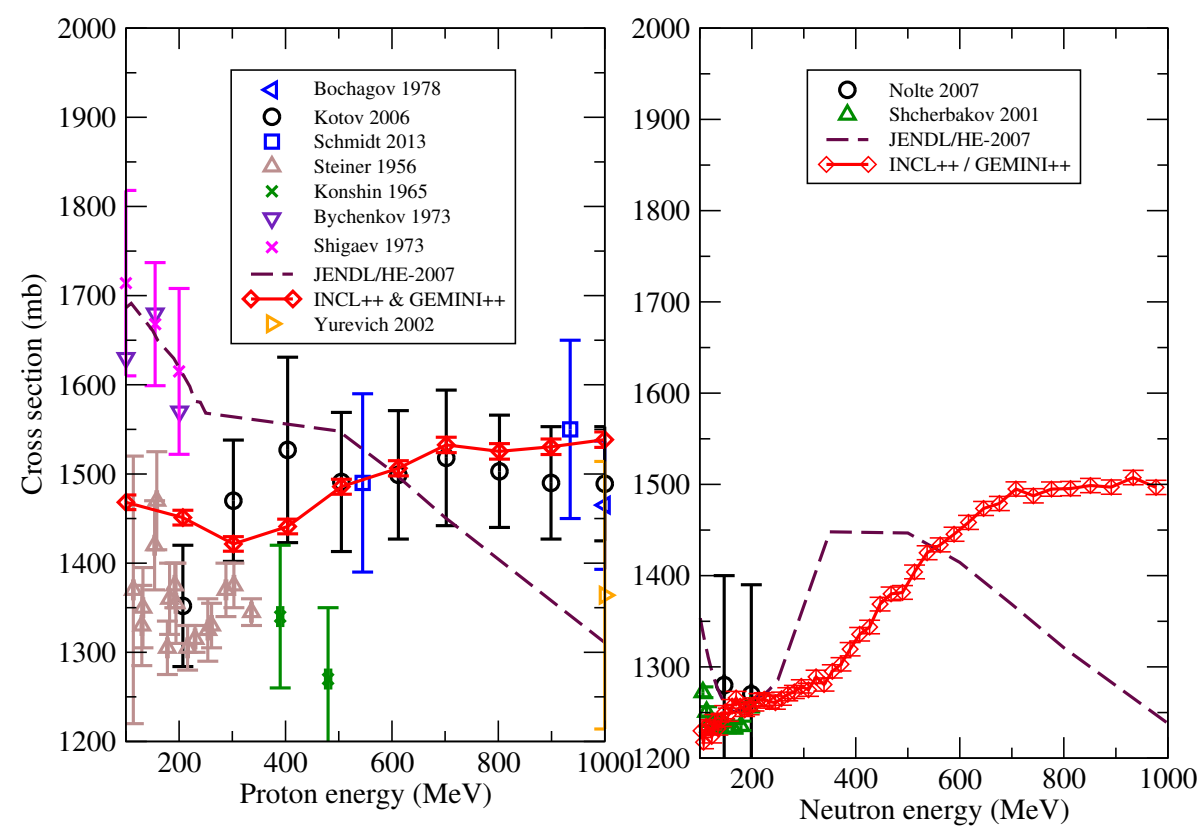

Figure 2: ${ }^{238} U$ fission cross sections. Experimental data are discussed in the text.

2013), obtained in an inverse-kinematics experiment, at 545 and $935 \mathrm{MeV}$, as well as with the older value at $1 \mathrm{GeV}$ from Ref. [45] (Bochagov 1978). On the contrary, the JENDL/HE-2007 evaluation is consistent with Refs. [46], 44] (Shigaev 1973 and Bychenkov 1973), as well as with Kotov's data in the intermediate range, up to $600 \mathrm{MeV}$, and the value at $1 \mathrm{GeV}$ from Ref. 39. (Yurevich 2002).

Experimental $(n, f)$ data are available below $200 \mathrm{MeV}$ : as already mentioned in connection with ${ }^{235} U$, the data of Ref.[41] can be considered as a true absolute cross section, while the data of Ref. [48] (Shcherbakov 2001) are normalized to the ${ }^{235} U(n, f)$ cross section recommended in Ref.[49]. We are in good agreement with Ref.[41, while underestimating the data of Ref.[50] (Prokofiev 1996) and overestimating the old data[51] (Goldanskii 1955). Again, our calculations are done with the same parameters for the $(p, f)$ and $(n, f)$ reactions, namely $\tilde{a}_{f} / \tilde{a}_{n}=1.038$ and $\Delta B_{f}=-0.3 \mathrm{MeV}$. 


\subsection{4 ${ }^{n a t} U$}

As shown in Figs. (1 2), the JENDL/HE-2007 evaluations of fission cross sections decrease with increasing projectile energy much faster than our calculations: a possible explanation for the discrepancy is that in the JENDL evaluations the energy dependence of fission cross sections is not derived from a full numerical simulation, but estimated by an analytical formula [52] with parameters based on systematics of experimental data, which are mainly available below $200 \mathrm{MeV}$. It is then of interest to check whether experimental indications of either trend exist at energies between $1 \mathrm{GeV}$ and $3 \mathrm{GeV}$, which is at the same time the upper limit of JENDL/HE-2007 evaluations and of the validity of the intranuclear cascade model in the version of INCL ++ we use. This possibility is provided by natural uranium, not explicitly evaluated in the JENDL library, but expected, in this range of projectile energy, to be very similar to ${ }^{238} \mathrm{U}$, its main component, with an abundance of more than 99\%. The left panel of Fig. 3 shows the ${ }^{\text {nat }} U(p, f)$ cross section calculated between $100 \mathrm{MeV}$ and $3 \mathrm{GeV}$ with the same parameters as ${ }^{238} U$, namely $\tilde{a}_{f} / \tilde{a}_{n}=1.038$ and $\Delta B_{f}=-0.3 \mathrm{MeV}$.

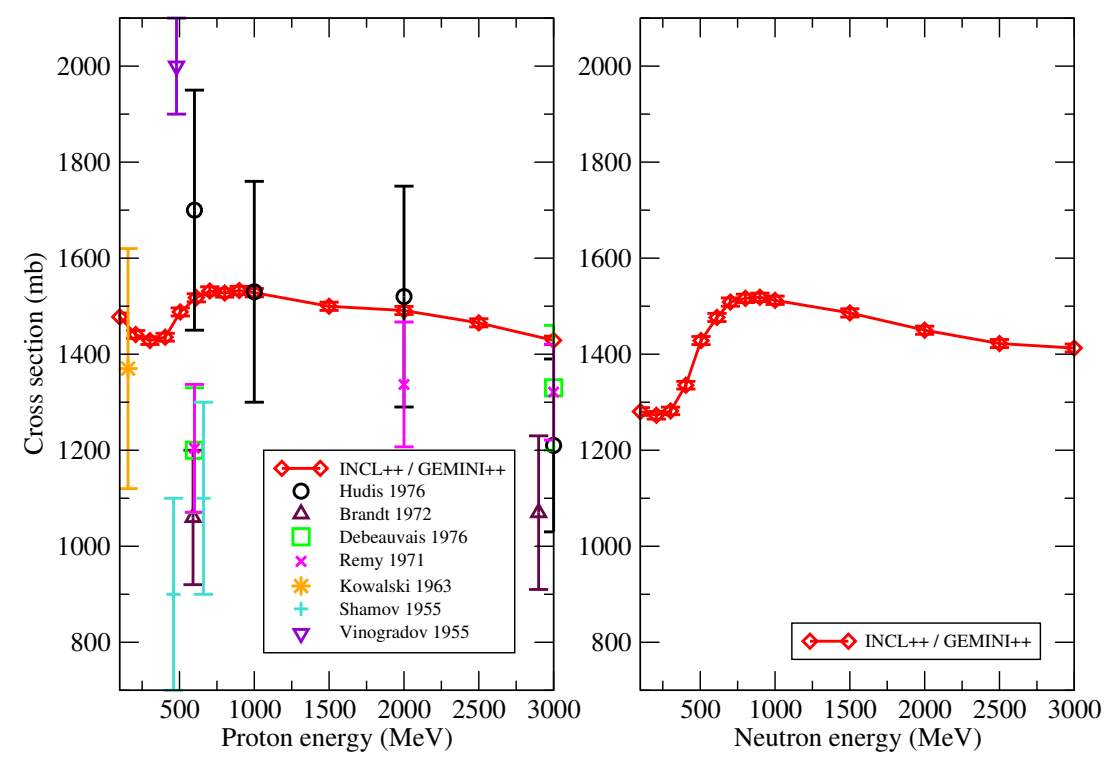

Figure 3: ${ }^{n a t} U$ fission cross sections. Experimental data are discussed in the text.

Our calculation compares well with the experimental data of Ref. 53] 
(Hudis 1976), which cover the full energy range, and is in marginal agreement with the point at $3 \mathrm{GeV}$, where, on the other hand, our calculation agrees with the data from Ref.[54] (Debeauvais 1976), Ref.[55] (Remy 1971) and Ref.[57] (Shamov 1955); in turn, the last three experiments are lower than Hudis 1976 and our calculations at lower energies. We are also in agreement with the point from Ref.[56] (Kowalski 1963).

Finally, all the data from Ref.[37] (Brandt 1972) are lower than our calculations, while the old experiment of Ref.[59] is much larger. Summing up, we believe that our calculations of the ${ }^{n a t} U(p, f)$ reactions are supported by experimental evidence up to $3 \mathrm{GeV}$. For the sake of completeness, the right panel of Fig. 3 shows the ${ }^{n a t} U(n, f)$ cross section, calculated with the same model parameters.

\subsection{5 $\quad{ }^{233} U$}

The only experimental data of the ${ }^{233} U(p, f)$ reaction in the energy range of interest are those of Kotov[5], shown in the left panel of Fig.4 together with our theoretical fit, which underestimates the two points at 404 and $505 \mathrm{MeV}$. In order to reproduce them, one should resort to an abnormally high value of the $\tilde{a}_{f} / \tilde{a}_{n}$ ratio, such as 1.6 or more. We have preferred to assume $\tilde{a}_{f} / \tilde{a}_{n}$ $=1.10$, higher than the values adopted for ${ }^{235} U$ and ${ }^{238} U$, but of the same order as that of the original fit of Ref.[5], which, in addition, points out the difficulty in reproducing $(p, f)$ cross sections of the uranium chain with their own cascade-exciton-fission model. The price we pay for this reasonable value of $\tilde{a}_{f} / \tilde{a}_{n}$ is a large reduction of the liquid-drop fission barriers of remnants, $\Delta B_{f}=-0.5 \mathrm{MeV}$.

The ${ }^{233} U(n, f)$ cross section relative to ${ }^{235} U$ has been measured at $\mathrm{n}_{-}$TOF up to $E_{n} \simeq 1 \mathrm{GeV}$, but final data are not yet available. Other relative $(n, f)$ measurements in a smaller energy range are those of Refs. [48, [61]. The right panel of Fig 4 shows that available $(n, f)$ data can be reproduced by a model calculation with $\tilde{a}_{f} / \tilde{a}_{n}=1.10$, as for the $(p, f)$ reaction, but $\Delta B_{f}=0$. A more detailed comparison of experimental and theoretical cross section ratios will be shown in the following sub-section.

\subsection{6 $\quad{ }^{232} \mathrm{Th}$}

As shown the left panel of Fig.5, the experimental results available for the $(p, f)$ cross section of ${ }^{232} \mathrm{Th}$ can be classified in two series: our reference 

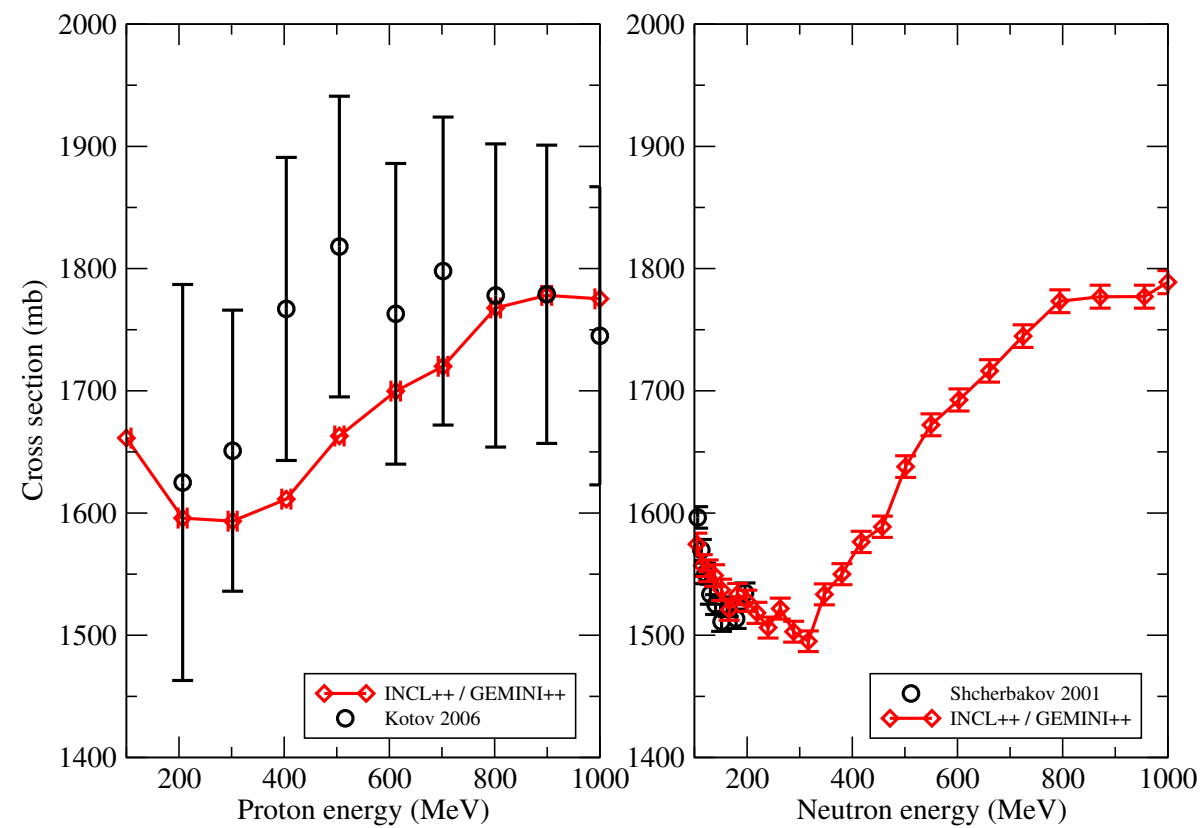

Figure 4: ${ }^{233} U$ fission cross sections. Experimental data are discussed in the text.

experiment[5], Ref.[45] (Bochagov 1978) and Ref.[58] (Saint-Laurent 1984) on one side and, on the other side, the majority of older experiments, with the exception of Ref.[59] (Vinogradov 1955), but including also the recent measurement 60. (Wenger 2006) at $590 \mathrm{MeV}$, which yield systematically lower values. Our choice between the two series of $(p, f)$ measurements has been motivated also by considerations on the experimental $(n, f)$ cross section, which is expected to be significantly smaller than the $(p, f)$ cross section in the $100 \mathrm{MeV}$ range: in particular, formula (6), based on the systematics of Ref.[34], predicts for ${ }^{232} T h \sigma_{p f} / \sigma_{n f}=1.55$ at $E_{\text {proj }} \simeq 180 \mathrm{MeV}$. A look at the experimental $(n, f)$ cross sections of Ref. 63] (Paradela 2006) and Ref. 48] (Shcherbakov 2001) induces us to prefer the series of higher experimental values [5] [58], otherwise the $(p, f)$ and $(n, f)$ cross sections at low energy would be more or less of the same order.

It is to be stressed, however, that both $(n, f)$ measurements [48] [63] are relative to ${ }^{235} U$ and that absolute cross sections have been obtained with different criteria of normalization; moreover, the $\mathrm{n}_{-} \mathrm{TOF}$ data 63] are still preliminary and subject to possible changes. As a consequence, the present 

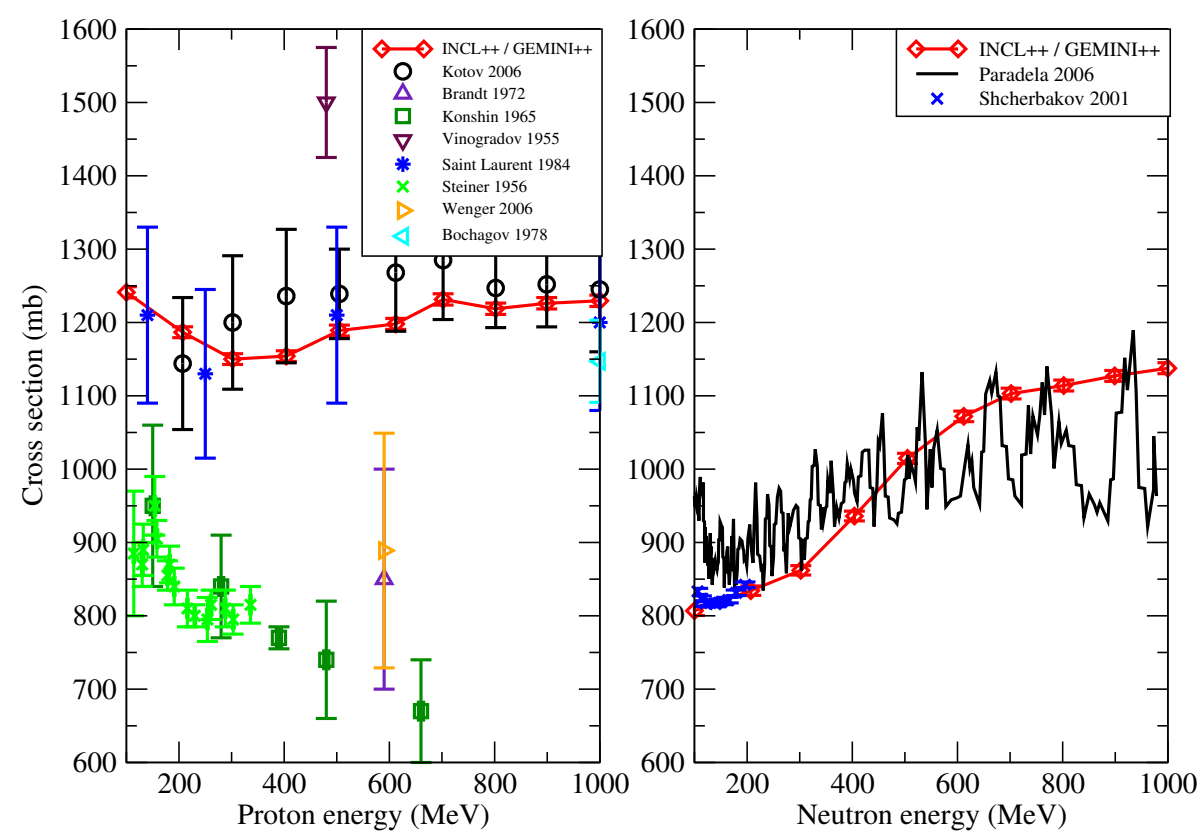

Figure 5: ${ }^{232}$ Th fission cross sections. Experimental data are discussed in the text.

calculation of the $(n, f)$ cross section should be taken as preliminary, too. The $(p, f)$ cross section has been reproduced with $\tilde{a}_{f} / \tilde{a}_{n}=1.040$ and $\Delta B_{f}=-0.3$ $\mathrm{MeV}$, the $(n, f)$ cross section with the same ratio of level density parameters, but with $\Delta B_{f}=+0.1 \mathrm{MeV}$, which is a relatively large discrepancy. If final $(n, f)$ values from the $\mathrm{n}_{-}$TOF experiment turn out to be higher than the preliminary ones, the discrepancy will be hopefully reduced.

\subsection{7 $\quad{ }^{237} N p$}

The basic measurement of the ${ }^{237} N p(p, f)$ cross section in the energy range of interest is again Kotov 2006 [5], to which one can add a point at $590 \mathrm{MeV}$ from Ref. 60] (Wenger 2006), a point at $660 \mathrm{MeV}$ from Ref.65] (Karapetyan 2009) and a point from Ref. 39] (Yurevich 2002) at $1 \mathrm{GeV}$; all of them agree with Kotov's data within the experimental uncertainties. The theoretical fit shown in the left panel of Fig. 6 is characterized by a large $\chi_{0}^{2}$ value, mainly because of the point at $207 \mathrm{MeV}$, which is largely overestimated by the calculation. . A similar problem appears in the original fit of Ref.[5], which reproduces data below $100 \mathrm{MeV}$ from other experiments, clearly not 

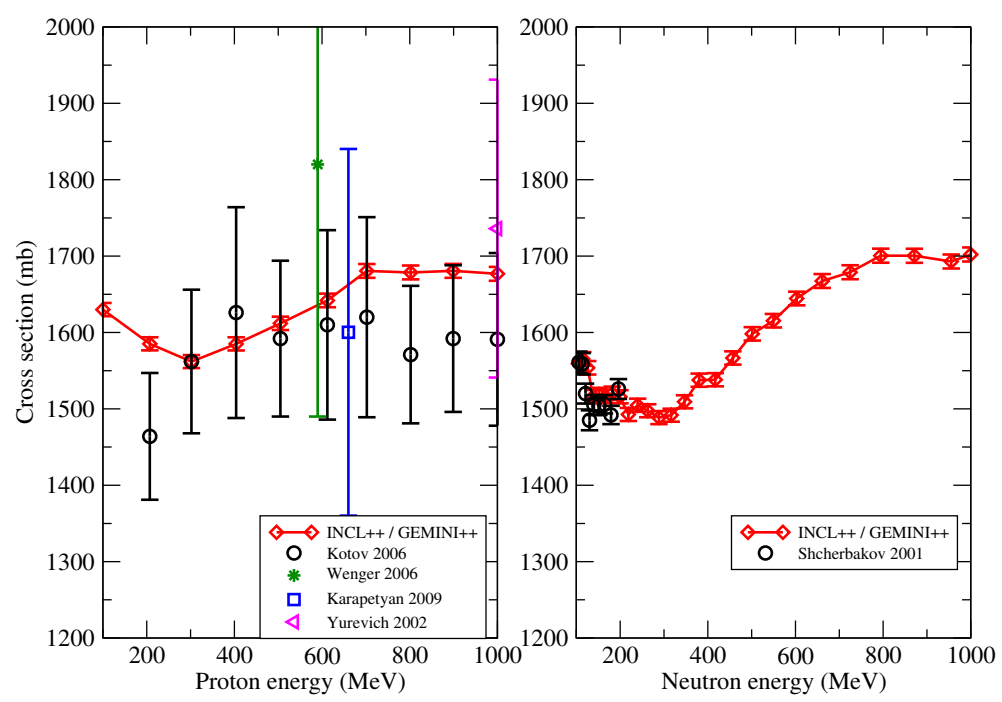

Figure 6: ${ }^{237} N p$ fission cross sections. Experimental data are discussed in the text.

compatible with the low value of the $(p, f)$ cross section at $207 \mathrm{MeV}$ Using the same model parameters, $\tilde{a}_{f} / \tilde{a}_{n}=1.040$ and $\Delta B_{f}=-0.2 \mathrm{MeV}$, for the ${ }^{237} N p(n, f)$ reaction, one obtains the cross section shown in the right panel of Fig.6, which reproduces the data of Ref. 48] (Shcherbakov 2001) below $200 \mathrm{MeV}$. It is to be recalled, however, that the measurements of Ref. [48] are relative to ${ }^{235} U(n, f)$ and that other measurements of relative cross sections exist, including those from n_TOF [9], which cover the full energy range of interest: they deserve a more detailed description in the following sub-section.

\subsection{8 $\quad{ }^{239} \mathrm{Pu}$}

The fit of the ${ }^{239} \mathrm{Pu}(p, f)$ cross section, for which the only data in the energy range of interest are those of Kotov [5], is characterized by a large $\chi_{0}^{2}$ value, because the two points at $207 \mathrm{MeV}$ and $302 \mathrm{MeV}$ are largely overestimated by our calculations, as shown in the left panel of Fig. 7.

However, the same problem exists in the original fit of Ref.[5], which clearly shows that a simultaneous reproduction of the two mentioned data and of those below $100 \mathrm{MeV}$ from other experiments is not possible. The same difficulty had already been encountered for the ${ }^{237} N p(p, f)$ reaction.

Using the same model parameters, $i$. e. $\quad \tilde{a}_{f} / \tilde{a}_{n}=1.036$ and $\Delta B_{f}=$ 

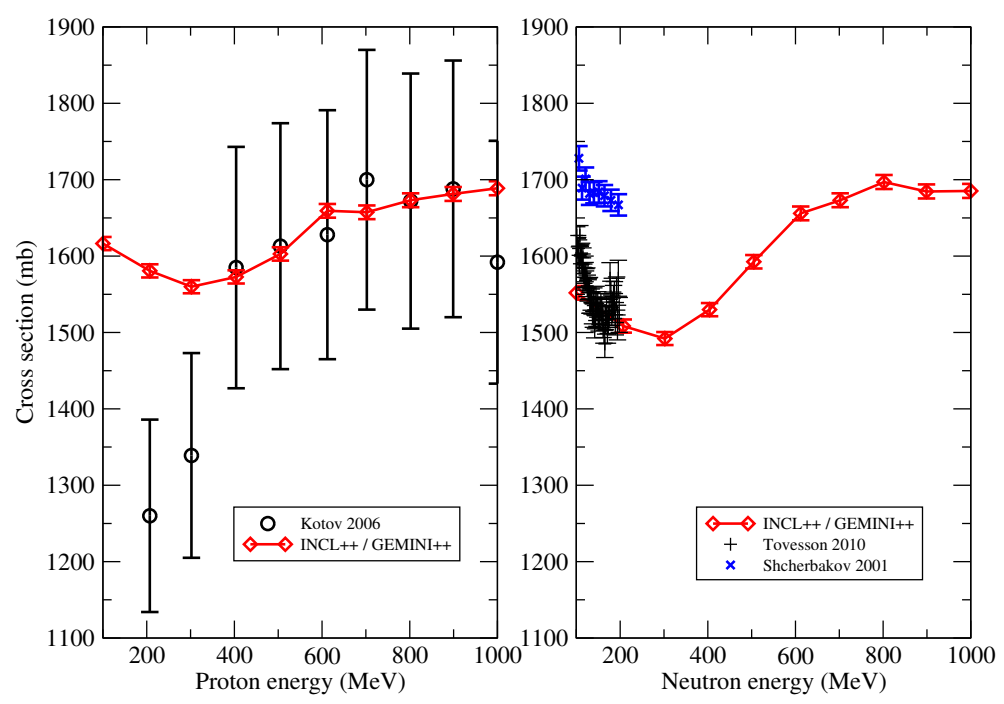

Figure 7: ${ }^{239} \mathrm{Pu}$ fission cross sections. Experimental data are discussed in the text.

$+0.3 \mathrm{MeV}$, in the $(n, f)$ calculations, shown in the right panel of Fig. 7 . yields a decent reproduction of the data of Ref. [67] (Tovesson 2010), while underestimating those of Ref. [48] (Shcherbakov 2001). It is to be stressed, however, that both measurements are relative to the ${ }^{235} U(n, f)$ reaction and that the experimental ratios have been normalized with different ${ }^{235} U(n, f)$ cross sections. In addition, the $(n, f)$ cross section of Ref. 48] appears to be larger than any reasonable extrapolation of the $(p, f)$ cross section down to the same energies, which is not expected on physical grounds. In any case, it is more meaningful to compare relative cross sections, as shown in the following sub-section.

\subsubsection{Lead - Bismuth}

In the lead-bismuth region, which is also very important for technological applications and is considered in the rest of this section, we have preferred to couple INCL ++ with ABLA07, since the latter appears to produce more accurate cross section fits than GEMINI++. The fission model parameters we have modified are the level density parameter at the saddle point, $a_{f}$, multiplied by a scale factor $k_{f}$, and the height of the liquid-drop fission barriers, shifted by a positive or negative quantity, $\Delta B_{f}$, as before. Table 2 
shows adopted parameters and $\chi^{2}$ tests for proton induced fission of ${ }^{208} \mathrm{~Pb}$, ${ }^{n a t} \mathrm{~Pb}$ and ${ }^{209} \mathrm{Bi}$.

\begin{tabular}{|c|c|c|c|c|c|}
\hline Isotope & $Z^{2} / A$ & $k_{f}$ & $\Delta B_{f}(\mathrm{MeV})$ & $\chi_{0}^{2}$ & $Q\left(\chi_{0}^{2}\right)$ \\
\hline${ }^{208} \mathrm{~Pb}$ & 32.33 & 1.0 & 0.0 & 6.21 & 0.52 \\
\hline${ }^{n a t} \mathrm{~Pb}$ & 32.45 & 0.995 & +0.16 & 20.04 & 0.0055 \\
\hline${ }^{209} \mathrm{Bi}$ & 32.96 & 1.01 & +0.18 & 2.53 & 0.93 \\
\hline
\end{tabular}

Table 2: Model parameters and $\chi^{2}$ tests for proton-induced fission of lead and bismuth.

The energy dependence of fission cross sections of lead and bismuth is quite different from that in the actinide region: at low energy, let us say $100 \mathrm{MeV}$, the $(n, f)$ cross section is 2 to 3 times smaller than the $(p, f)$ cross section and neither of them reaches a plateau at high energy, $1 \mathrm{GeV}$. Therefore, knowledge of the $(p, f)$ cross section in this energy range yields less useful information on the $(n, f)$ cross section with respect to actinides.

\subsubsection{0 $\quad{ }^{208} \mathrm{~Pb}$}

An experiment performed at the institute of Ref. [5] produced the ${ }^{208} \mathrm{~Pb}(p, f)$ cross section at the same energies and the results, published in Ref. 68 (Vaishnene 2010), are shown in the left panel of Fig,8, together with our calculations, which reproduce them satisfactorily with the default parameters of the ABLA07 model, $i . e . k_{f}=1$ and $\Delta B_{f}=0$.

It is to be pointed out that $(p, f)$ data below $200 \mathrm{MeV}$ are consistent with Ref.668], with the exception of Ref.[69] (Flerov 1972), whose data are somewhat lower than our calculation. At high energy, on the contrary, the recent result of Ref.44] (Schmidt 2013) at $500 \mathrm{MeV}$ as well as the value of Ref.[70] (Enqvist 2001) at $1 \mathrm{GeV}$ are higher. Both experiments [44] [70] were performed in inverse kinematics. New data in this energy interval are needed in order to clarify the situation.

$(n, f)$ data are available up to $200 \mathrm{MeV}$ from Refs.[71] (Smirnov 2004) and 72 (Rhyzov 2006), which are in mutual agreement. They are reproduced in the right panel of Fig. 8 with the default value of $\tilde{a}_{f}$, at the cost of increasing the fission barriers by a quantity $\Delta B_{f}=0.3 \mathrm{MeV}$. 

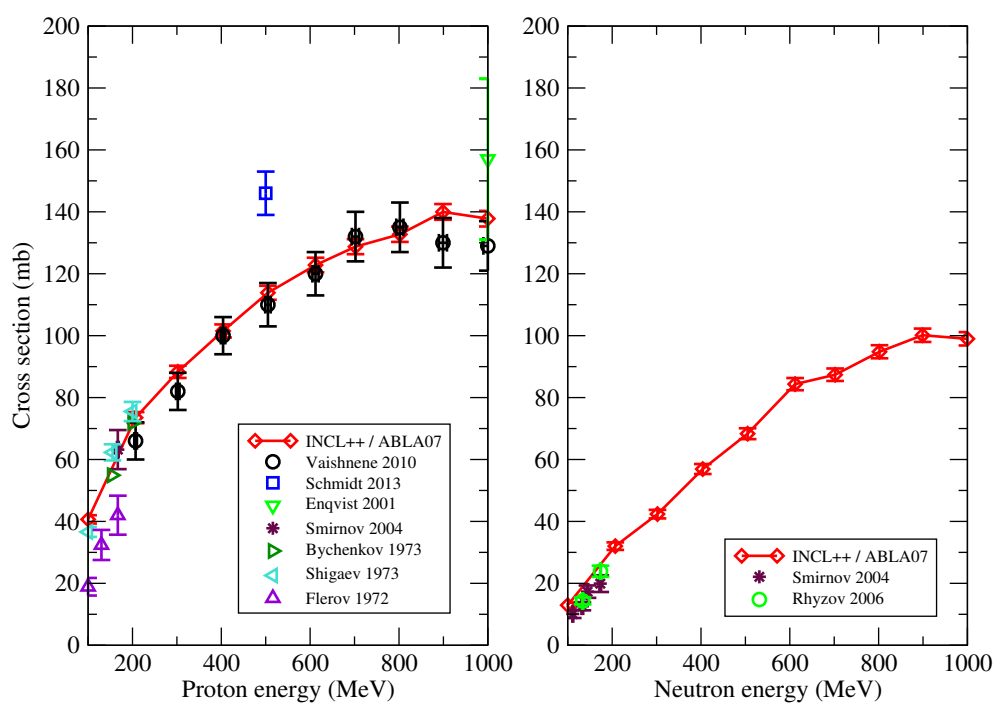

Figure 8: ${ }^{208} \mathrm{~Pb}$ fission cross sections. Experimental data are discussed in the text.

\subsubsection{1 ${ }^{\text {nat }} \mathrm{Pb}$}

Fission of natural lead is very important for applications to accelerator-driven systems, where the eutectic system acting as a spallation target and as a coolant consists of ${ }^{n a t} \mathrm{~Pb}$ and ${ }^{209} \mathrm{Bi}$. As usual, Kotov [5] provides $(p, f)$ data from $200 \mathrm{MeV}$ to $1 \mathrm{GeV}$. An older measurement (Flerov 1972)[69] below $200 \mathrm{MeV}$ appears to be compatible with Kotov's data[5], while other old experiments[38, [37], 55] (Koshin 1965, Brandt 1972 and Remy 1971, respectively) at higher energies seem to suggest a systematically higher cross section. As shown by the corresponding $\chi^{2}$ test in Table 2 , our fit to the $(p, f)$ data of Kotov [5], obtained by reducing the default $\tilde{a}_{f}$ parameter by a factor $k_{f}=0.995$ and by reducing the fission barrier by a quantity $\Delta B_{f}=-0.16$ $\mathrm{MeV}$ is not very satisfactory; in particular, our calculations overestimate the low energy points, as shown in the left panel of Fig 9 .

The same difficulty appears also in $(n, f)$ calculations, carried out with $k_{f}=1.005$ and $\Delta B_{f}=0$, which overestimate the experimental data below $200 \mathrm{MeV}$, where there is good agreement of experiments (Prokofiev 1996) [50], (Shcherbakov 2001) 48, (Smirnov 2004)[71], (Nolte 2007) 41], as shown in the right panel of Fig. 9. The relative $(n, f)$ data measured at $\mathrm{n}_{-}$TOF up to $1 \mathrm{GeV}[10]$ will be discussed in the next sub-section. 

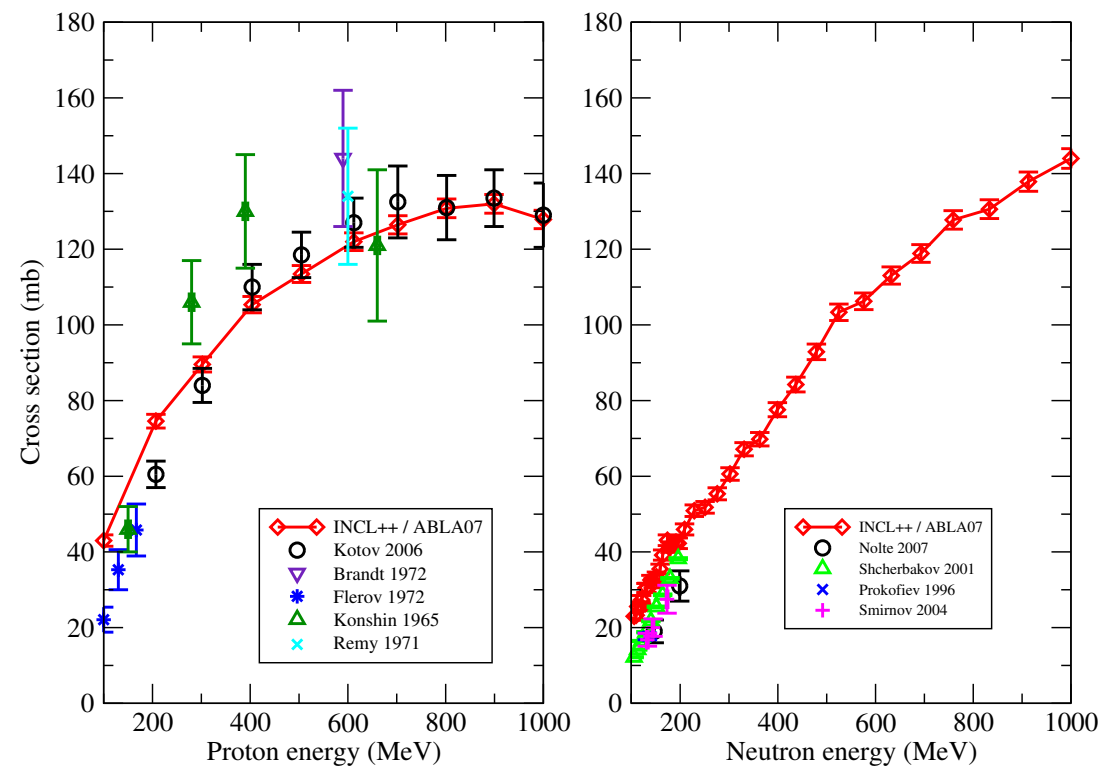

Figure 9: ${ }^{n a t} \mathrm{~Pb}$ fission cross sections. Experimental data are discussed in the text.

\subsubsection{2 ${ }^{209} B i$}

As far as the $(p, f)$ cross section is concerned, several measurements exist for ${ }^{209} \mathrm{Bi}$ and the majority of them (Steiner 1956) [36], (De Carvalho 1962) [73], (Konshin 1965) [38], (Brandt 1972) [37], (Bychenkov 1973) 47], (Debeauvais 1976) 54 are in reasonable agreement with our reference experiment Kotov 2006 [5], as shown in the left panel of Fig.10, the main exceptions are the point at $1 \mathrm{GeV}$ from Ref. 45] (Bochagov 1978), which is much lower than the corresponding point from Ref.[5], as well as Ref.[53] (Hudis 1976), whose data are systematically higher than those of Ref.[5]. The latter are well reproduced in our calculations by multiplying the default $\tilde{a}_{f}$ parameter by a factor $k_{f}=1.01$ and increasing the fission barriers by an amount $\Delta B_{f}=+0.18$ $\mathrm{MeV}$. Among the $(n, f)$ experiments, a key role is played by Refs. [50, [41] (Prokofiev 1996, Nolte 2007), which are relative to $(n, p)$ scattering and can be considered as measurements of the absolute $(n, f)$ cross section, while Refs 48] (Shcherbakov 2001), [74] (Fomichev 2004), [75] (Laptev 2007), [10] (Tarrío 2011) are relative to ${ }^{235} U(n, f)$ and Refs. [71,,[72] (Smirnov 2004 and Rhyzov 2006, respectively), are relative to ${ }^{238} U$. Only Refs. [10], [74] extend above $200 \mathrm{MeV}$. Using in $(n, f)$ calculations the same parameters adopted in 

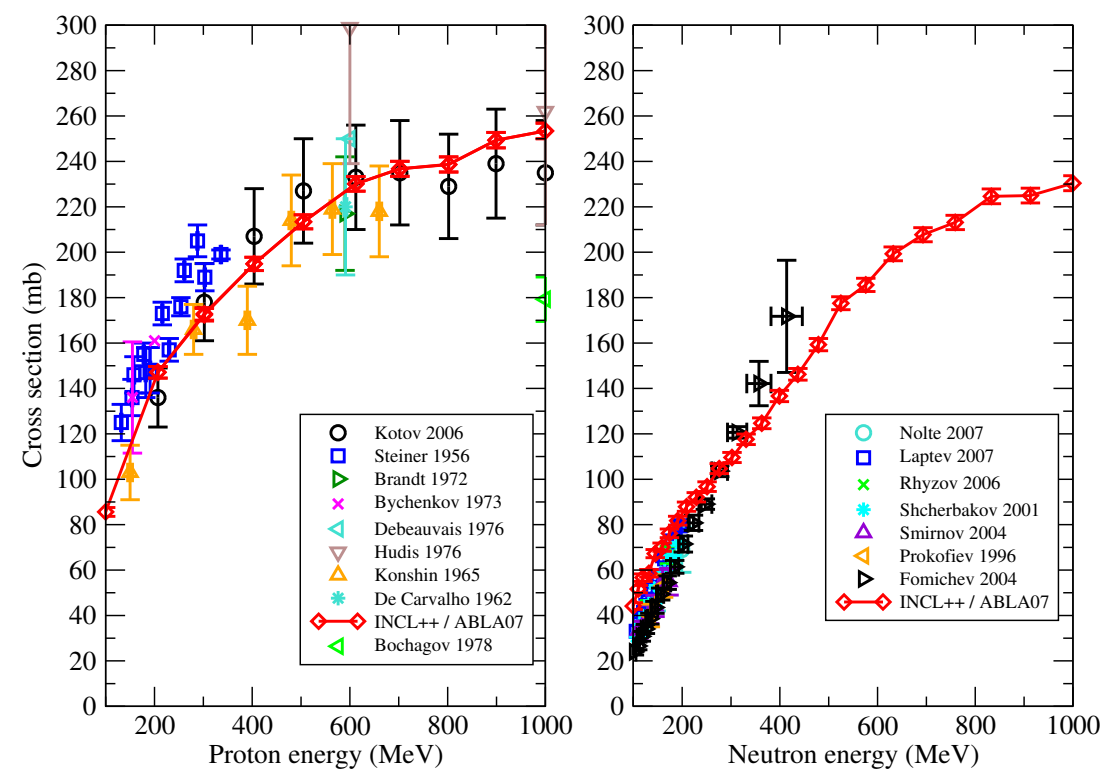

Figure 10: ${ }^{209} \mathrm{Bi}$ fission cross sections. Experimental data are discussed in the text.

fitting $(p, f)$ data would underestimate the data of Ref.[74] from 200 to 400 $\mathrm{MeV}$, while reproducing the data of Ref.[10] relative to ${ }^{235} U$ reasonably well. As a compromise between the two sets of data, we have adopted in $(n, f)$ calculations $k_{f}=1.01$ and $\Delta B_{f}=0$. More details will be given in the next sub-section.

\subsection{Relative Cross Sections}

This sub-section is dedicated to $(n, f)$ measurements relative to the ${ }^{235} U(n, f)$ reaction, taken as a standard, even if no reliable data of its absolute cross section exist above $200 \mathrm{MeV}$, with particular reference to $\mathrm{n}_{-} \mathrm{TOF}$ 6] measurements up to $1 \mathrm{GeV}$. For those cases [9] [10] where the experimental ratios measured at $\mathrm{n}_{-}$TOF have been normalized in the original publications to the ${ }^{235} U(n, f)$ cross section from the JENDL/HE-2007 library a comparison of absolute cross sections has been added to that of relative cross sections. The parameters adopted in the calculations are those given for the $(n, f)$ reactions in the sub-section on absolute cross sections.

The cross section ratio to be compared with measurements is the ratio of 
theoretical cross sections obtained with our Monte Carlo simulations, which make it possible to associate to any $\sigma(E)$ an uncertainty $\Delta \sigma(E)$ attributable to counting statistics. If $R=\sigma_{1} / \sigma_{2}$ is the ratio in question, the relative error $\Delta R / R$ is obtained by the quadratic law of propagation of relative errors of two uncorrelated variables $\sigma_{1}$ and $\sigma_{2}$

$$
\frac{\Delta R}{R}=\left[\left(\frac{\Delta \sigma_{1}}{\sigma_{1}}\right)^{2}+\left(\frac{\Delta \sigma_{2}}{\sigma_{2}}\right)^{2}\right]^{1 / 2} .
$$

\subsection{1 ${ }^{238} U$}

Figure 11 compares calculated and experimental ratios for the ${ }^{238} U(n, f)$ reaction.

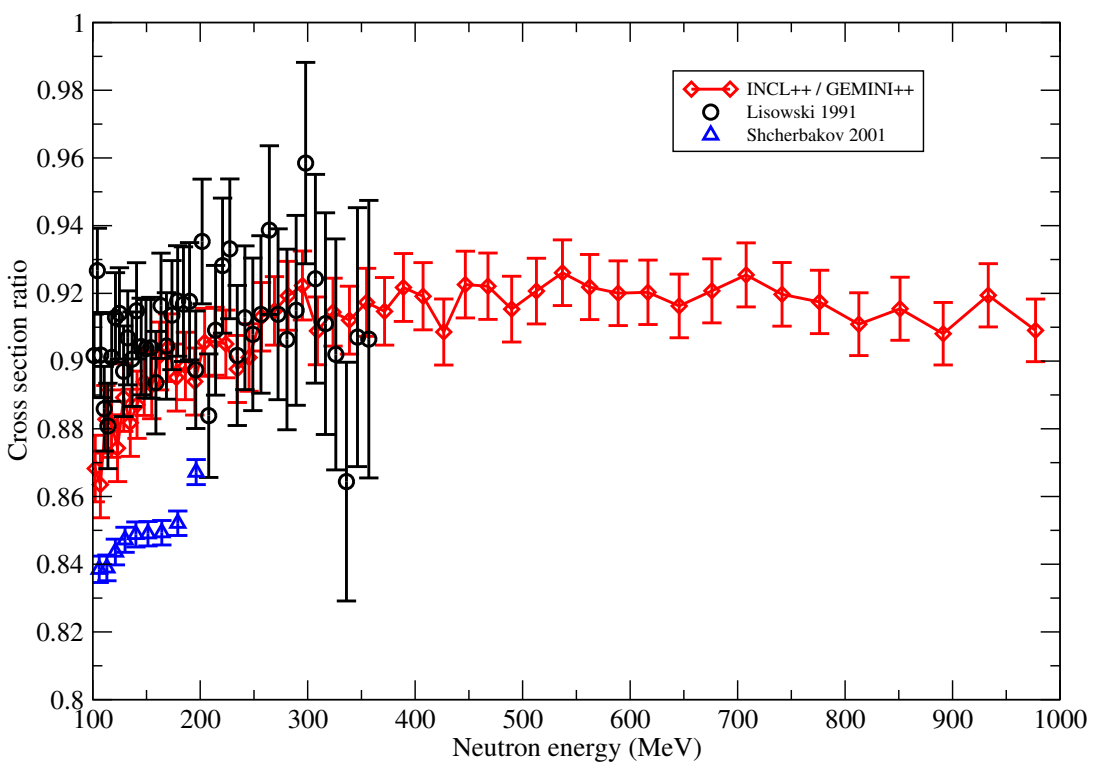

Figure 11: Ratio of ${ }^{238} U(n, f)$ to ${ }^{235} U(n, f)$ fission cross section. Experimental data are discussed in the text.

Our results agree with Ref.[40], which still represents one of the most reliable measurements of $(n, f)$ cross sections in the intermediate energy region, and are somewhat higher than Ref.[48]. Preliminary $\mathrm{n}_{-}$TOF results up to 1 $\mathrm{GeV}$, not reported in Fig. 11, compare reasonably well with our calculations. 


\subsection{2 ${ }^{234} U$}

No $(p, f)$ data are available for ${ }^{234} U$, whose $(n, f)$ cross section relative to ${ }^{235} \mathrm{U}$ has been measured up to $400 \mathrm{MeV}$ in Ref.661. (Lisowski $1991 \mathrm{~b}$ ) and at the $\mathrm{n}_{-} \mathrm{TOF}$ facility up to $1 \mathrm{GeV}$ using parallel plate avalanche counters in Ref.[9] (Paradela 2010) and up to $200 \mathrm{MeV}$ using fission ionization chambers in Ref.[62]; the two n_TOF experiments are in good mutual agreement, therefore we take into account only the more extended measurement [9] for comparison with our calculations, done with the same fission parameters as ${ }^{235} U$, namely $\tilde{a}_{f} / \tilde{a}_{n}=1.050$ and $\Delta B_{f}-0.2 \mathrm{MeV}$. The left panel of Fig. 12 compares theoretical and experimental ratios, in overall good agreement, while the right panel compares our absolute cross section with the one obtained in Ref. 9] by normalizing the experimental ratio to the JENDL/HE-2007 evaluation of the ${ }^{235} U(n, f)$ cross section. As one can see from the right panel
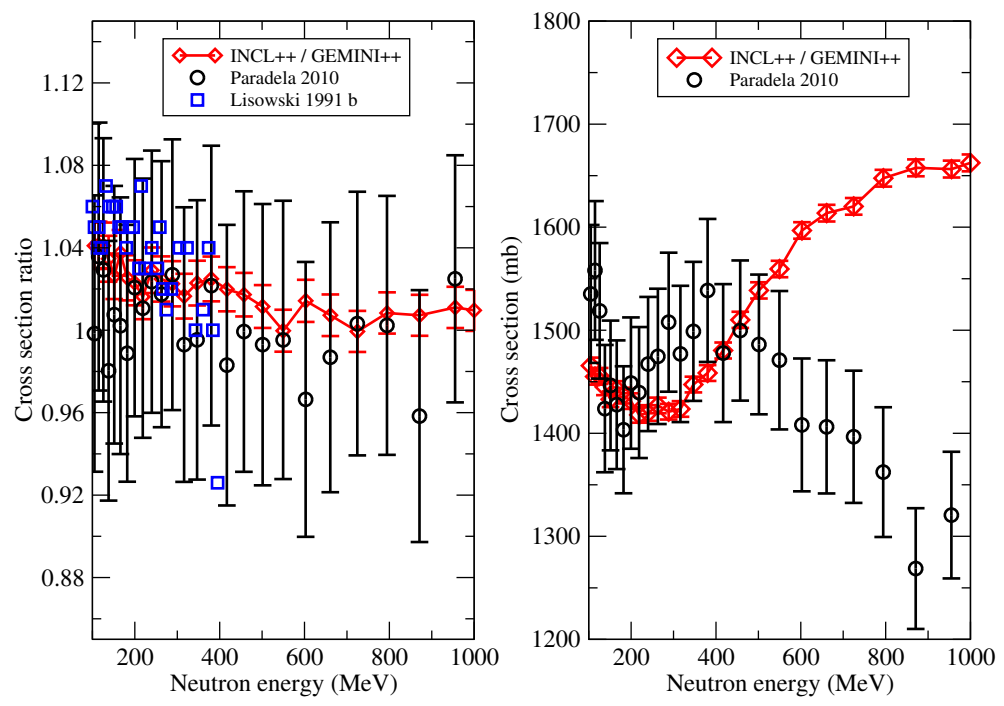

Figure 12: Left panel: $(n, f)$ cross section of ${ }^{234} U$ relative to ${ }^{235} U$. Right panel: absolute $(n, f)$ cross section of ${ }^{234} U$. Experimental data are discussed in the text.

of Fig. 1 the disagreement increases with increasing neutron energy, because our calculated ${ }^{235} U(n, f)$ cross section shows a large plateau at high energy, while the JENDL evaluation steadily decreases in the same energy range. 


\subsection{3 ${ }^{233} U$}

A somewhat larger value of $\tilde{a}_{f} / \tilde{a}_{n}$ is necessary for reproduction of the fission measurements of ${ }^{233} U$. Fig 13 shows a comparison of available data relative to ${ }^{235} U$ from Refs. 61, 48, with the theoretical cross section ratio obtained with $\tilde{a}_{f} / \tilde{a}_{n}=1.10$ and $\Delta B_{f}=0$. A positive value of $\Delta B_{f}$ would further bring the relative cross section to better agreement with experimental data. New data up to $1 \mathrm{GeV}$ are expected in the near future from the $\mathrm{n}_{-} \mathrm{TOF}$ experiment.

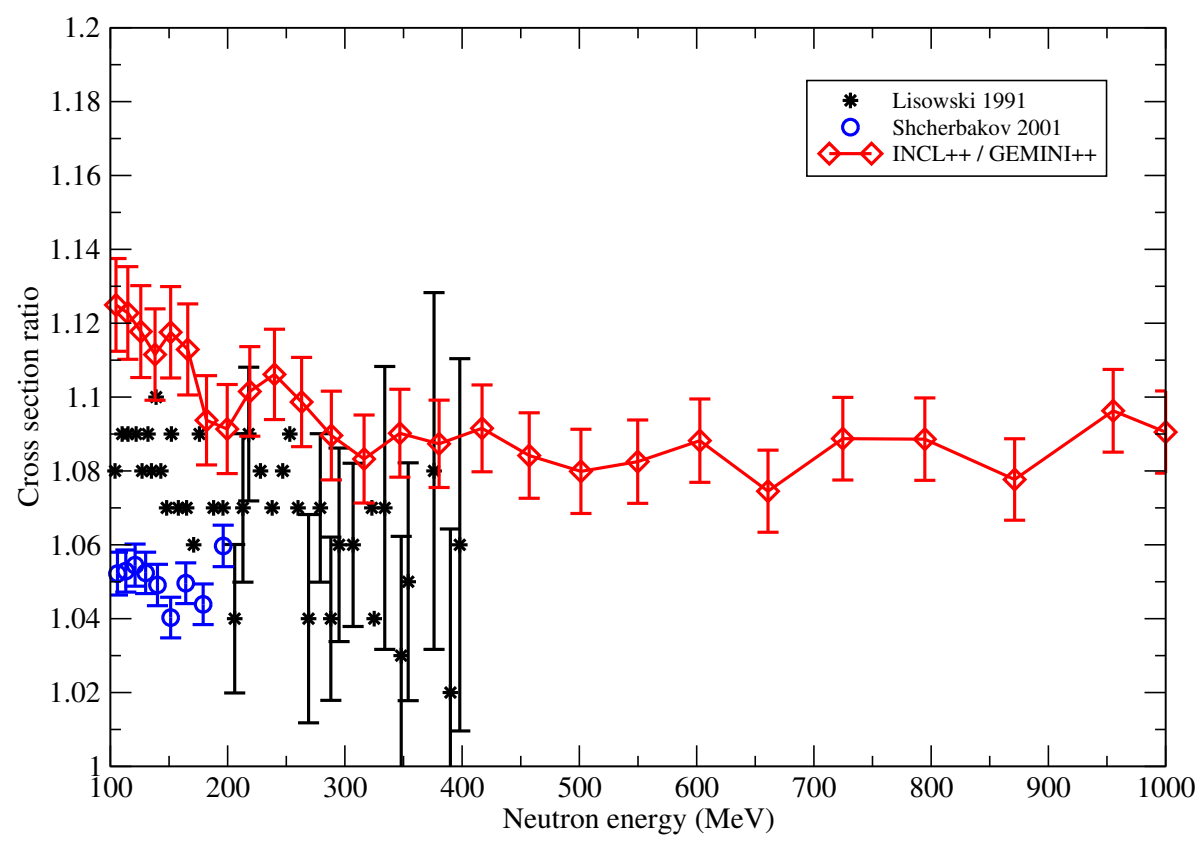

Figure 13: $(n, f)$ cross section of ${ }^{233} U$ relative to ${ }^{235} U$. Experimental data are discussed in the text.

\subsection{4 $\quad{ }^{232} T h$}

As already discussed in the preceding sub-section, the $\mathrm{n}_{-} \mathrm{TOF}$ results for the $(n, f)$ cross section of ${ }^{232} T h$ are still preliminary and a more reliable ${ }^{235} U(n, f)$ cross section in the intermediate energy range is recommended for their normalization. The difficulty with the present data[63] clearly appears in the comparison of our calculated cross-section ratio, which reproduces the $(n, f)$ values of Fig 5, with the presently available experimental ratios [64] 48], 
which are in reasonable mutual agreement and significantly lower than our results, as shown in Fig.14.

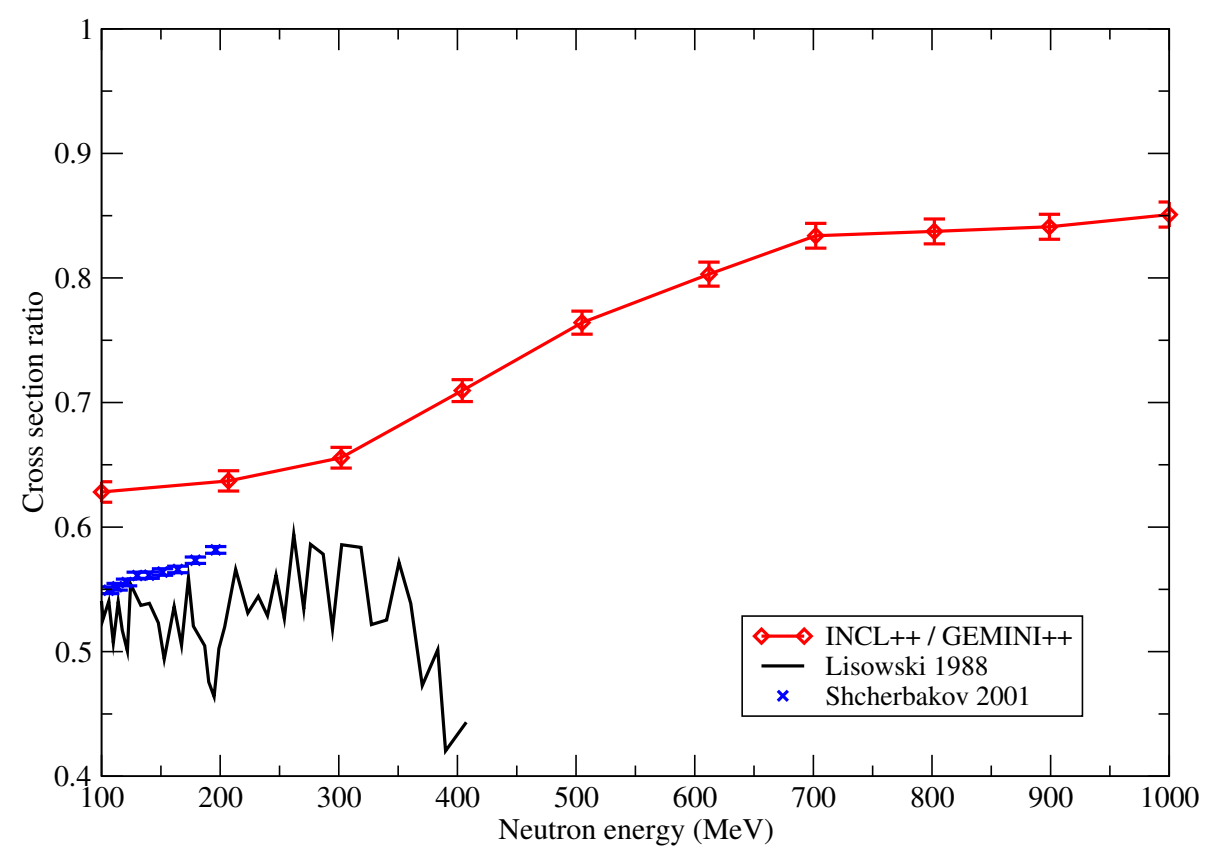

Figure 14: $(n, f)$ cross section of ${ }^{232} T h$ relative to ${ }^{235} U$. Experimental data are discussed in the text.

\subsection{5 $\quad{ }^{237} N p$}

The left panel of Fig. 15 compares the ratios of the ${ }^{237} N p(n, f)$ cross section to the ${ }^{235} U(n, f)$ cross section measured at $\mathrm{n}_{-}$TOF [9] with our calculated results, which turn out to be in overall agreement with the experiment. On the contrary, the right panel shows a significant disagreement of the corresponding absolute cross sections in the high energy region, owing to the normalization to the JENDL/HE-2007 evaluation of ${ }^{235} U(n, f)$ adopted in Ref.[9].

\subsection{6 $\quad{ }^{239} P u$}

The relative $(n, f)$ cross section of ${ }^{239} P u$, shown in Fig. 16, compares reasonably well with the data of Ref. [40] below $150 \mathrm{MeV}$ and with those of 

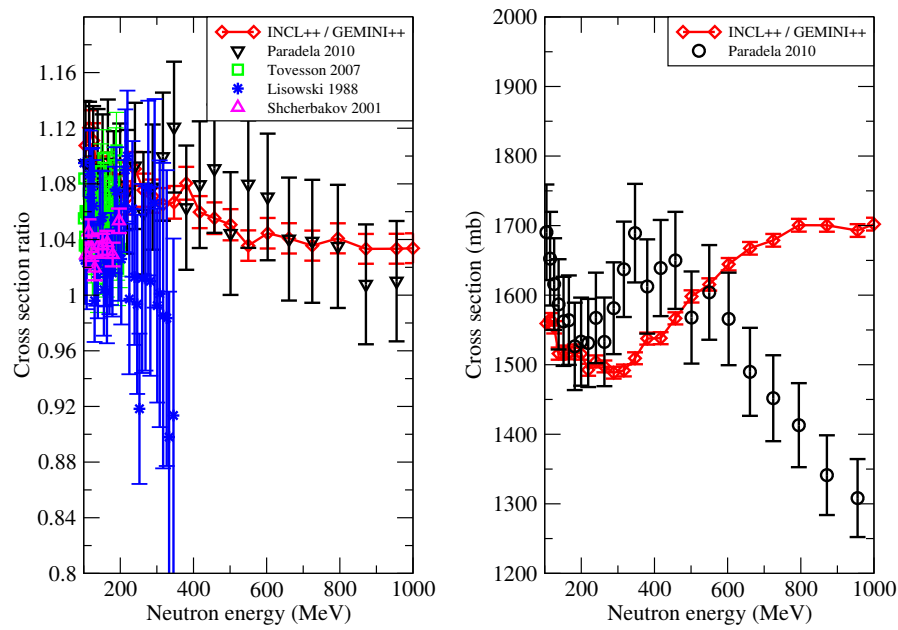

Figure 15: Left panel: $(n, f)$ cross section of ${ }^{237} N p$ relative to ${ }^{235} U$. Right panel: absolute $(n, f)$ cross section of ${ }^{237} N p$. Experimental data are discussed in the text.

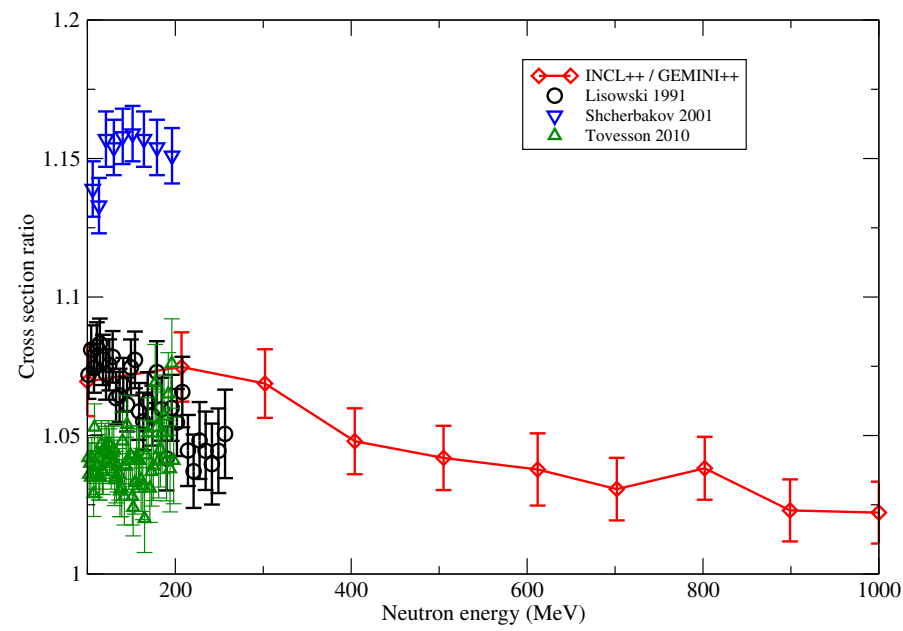

Figure 16: $(n, f)$ cross section of ${ }^{239} \mathrm{Pu}$ relative to ${ }^{235} \mathrm{U}$. Experimental data are discussed in the text.

Ref.67] between 150 and $200 \mathrm{MeV}$. The two series of data partially overlap, while those of Ref.[48] are systematically higher than them and than our calculations as well. 


\subsection{7 $\quad{ }^{n a t} \mathrm{~Pb}$}

The $(n, f)$ cross section of ${ }^{n a t} \mathrm{~Pb}$ relative to ${ }^{235} \mathrm{U}$ has been measured at $\mathrm{n}_{-} \mathrm{TOF}$ up to $1 \mathrm{GeV}[10]$. The left panel of Fig. 17 shows a comparison of experimental and theoretical cross section ratios, computed by increasing the default $a_{f}$ parameter by a factor of 1.005 , without any change of fission barriers. As already noticed for the absolute $(n, f)$ cross section discussed in the preceding sub-section, the calculations overestimate the experimental data at low energies. For the sake of completeness, the right panel of Fig. 17 compares the calculated absolute cross section with the one obtained by normalizing the relative cross section measured at $\mathrm{n}_{-} \mathrm{TOF}$ to the evaluated ${ }^{235} U(n, f)$ cross section of the JENDL/HE-2007 library. The disagreement at high energies is mainly due to the difference of the two computed ${ }^{235} \mathrm{U}$ cross sections.
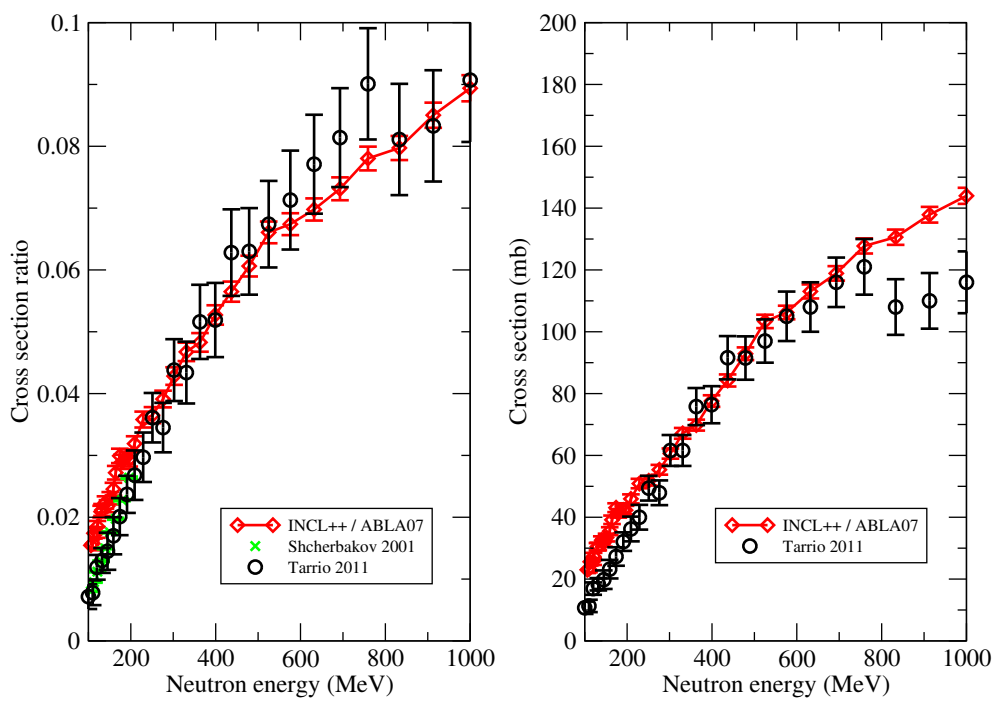

Figure 17: Left panel: $(n, f)$ cross section of ${ }^{n a t} \mathrm{~Pb}$ relative to ${ }^{235} \mathrm{U}$. Right panel: absolute $(n, f)$ cross section of ${ }^{n a t} P b$. Experimental data are discussed in the text.

\subsection{8 $\quad{ }^{209} \mathrm{Bi}$}

Finally, Fig. 18 shows the results of our $(n, f)$ calculations for ${ }^{209} B i$, which slightly overestimate the data of Ref. [10] relative to ${ }^{235} \mathrm{U}$ below $200 \mathrm{MeV}$, as shown in the left panel of the figure. 

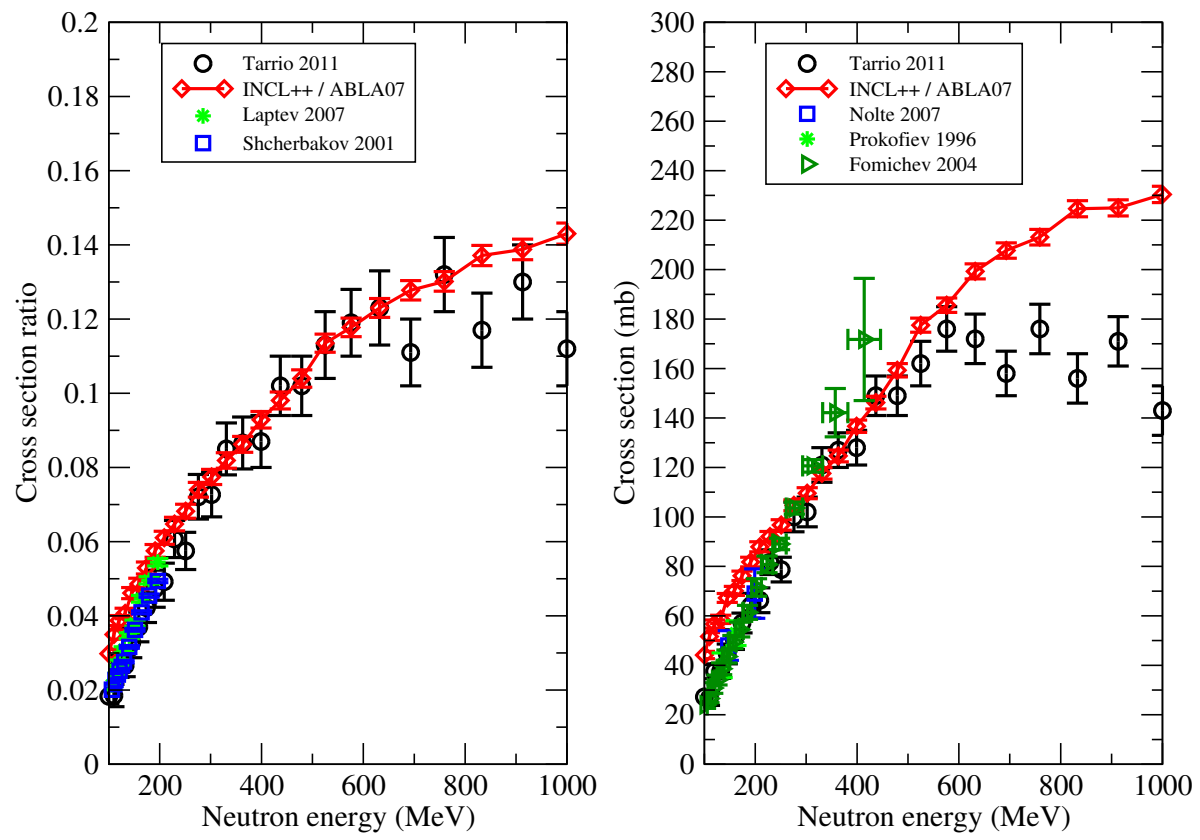

Figure 18: Left panel: $(n, f)$ cross section of ${ }^{209} B i$ relative to ${ }^{235} U$. Right panel: absolute $(n, f)$ cross section of ${ }^{209} B i$. Experimental data are discussed in the text.

This is the result of a compromise that takes into account the absolute data of Ref.[74] up to $400 \mathrm{MeV}$, shown in the right panel of the figure together with the relative data of Ref.[10] normalized to the JENDL/HE-2007 cross section of the ${ }^{235} U(n, f)$ reaction. Also the measurement of Ref. [74] is relative to ${ }^{235} U$, but we do not know the values adopted for normalization, so that we could consider only the absolute cross section. The disagreement with Ref.[10] above $500 \mathrm{MeV}$ is mainly due to the JENDL/HE-2007 cross section adopted in that reference for normalization.

Summing up, $(p, f)$ and $(n, f)$ cross sections have been reproduced with the same model parameters in the cases of ${ }^{235,238} \mathrm{U},{ }^{237} \mathrm{~Np},{ }^{239} \mathrm{Pu}$ and ${ }^{208} \mathrm{~Pb}$; the same level density parameters, but different corrections to the heights of fission barriers have been adopted for ${ }^{233} \mathrm{U},{ }^{232} \mathrm{Th}$ and ${ }^{209} \mathrm{~B} i$; finally, slightly different level density parameters and barrier height corrections have been used for ${ }^{n a t} \mathrm{~Pb}$ : this is a peculiar case, since the natural element consists of sizable abundances of ${ }^{208} \mathrm{~Pb},{ }^{207} \mathrm{~Pb}$ and ${ }^{206} \mathrm{~Pb}$. In principle, each isotope should have its own model parameters, but, for the sake of simplicity, we 
have chosen to treat ${ }^{n a t} \mathrm{~Pb}$ as an effective nucleus.

\section{Conclusions and Outlook}

In the present work we have reproduced $(p, f)$ cross sections in the intermediate energy range for a number of actinides and pre-actinides relevant to nuclear energy applications by a two-parameter fit: an increase of the level density parameter at the saddle point with increasing fissility parameter is observed in specific isotopic chains, such as uranium nuclei in Table I and lead nuclei in Table II, but the present data are not sufficient to determine a global trend in either region. Therefore, the results of the present work cannot be used for prediction of fission cross sections of isotopes for which neither $(p, f)$ or $(n, f)$ data exist in the intermediate energy range.

The model parameters that reproduce $(p, f)$ cross sections have been used as a first guess in the calculation of $(n, f)$ cross sections, where the comparison with experiments is complicated by the fact that the large majority of data are relative to fission standards, such as ${ }^{235,238} U$ in the actinide region and/or ${ }^{209} \mathrm{Bi}$ in the pre-actinide region, but even for the standards absolute cross section data are not available above $200 \mathrm{MeV} .(n, f)$ cross sections are systematically lower than $(p, f)$ cross sections around $100 \mathrm{MeV}$, but the differences are expected to reduce with increasing projectile energy, particularly for actinides, whose $(p, f)$ data exhibit a large plateau from $500 \mathrm{MeV}$ to 1 $\mathrm{GeV}$. This effect is clearly seen in our calculations.

As far as $(n, f)$ cross sections are concerned, the only relative measurements extending up to $1 \mathrm{GeV}$, due to the $\mathrm{n}_{-} \mathrm{TOF}$ collaboration, are satisactorily reproduced by our calculations. The disagreement between corresponding absolute cross sections is almost entirely due to the normalization to the JENDL/HE-2007 evaluation of the ${ }^{235} U(n, f)$ reaction adopted in $\mathrm{n}_{-}$TOF papers [9], 10].

A deeper comparison with evaluated data libraries such as JENDL/HE2007 would require an extension of the energy range of our calculations: as already shown in the case of ${ }^{n a t} U$, whose calculations up to $3 \mathrm{GeV}$ look promising, the codes used in this work allow us to extend cross section calculations up to $3 \mathrm{GeV}$, but moving the lower extremum down to $20 \mathrm{MeV}$ could only be done by resorting to fully quantum-mechanical models, since the very concept of intranuclear cascade model loses its applicability at low energies. 
A recent example of consistent calculations of $(p, f)$ and $(n, f)$ calculations in the energy range from $20 \mathrm{MeV}$ to $1 \mathrm{GeV}$ for a number of actinides is provided by Ref.[76], where use is made of the MCFx system of codes, permitting a good reproduction of many experimental data at the cost of adjusting the heights of the fission barriers of remnants obtained from microscopic calculations. This has been done by us, too, at the very phenomenologic level permitted by the $\Delta B_{f}$ parameter of Tables I-II.

An extension of our fission calculations to the energy range from $20 \mathrm{MeV}$ to $3 \mathrm{GeV}$ is planned for the nuclei already suggested as fission standards, ${ }^{235,238} U$ and ${ }^{209} B i$, with the specific scope of support to the analysis of $(n, f)$ cross sections measured at the $\mathrm{n}_{-}$TOF facility [6] at CERN.

\section{Acknowledgements}

We acknowledge valuable discussions with Drs. Sylvie Leray, Aleksandra Kelić-Heil, Nicola Colonna, Carlos Paradela and Laurent Tassan-Got.

\section{References}

[1] W. Maschek et al., Progr. Nucl. En. 50 (2008) 333.

[2] N. Colonna et al., Energy Environ. Sci. 3 (2010) 1910.

[3] A. I. Obukhov, Phys. Part. Nucl. 32 (2001) 162.

[4] A. V. Prokofiev, Nucl. Instr. Meth. Phys. Res. A 463 (2001) 557.

[5] A. A. Kotov et al., Phys. Rev. C 74 (2006) 034605.

[6] C. Guerrero et al. (the n_TOF Collaboration), Eur. Phys. J. A 49 (2013) 27.

[7] M. B. Chadwick et al., Nucl. Data Sheets 112 (2011) 2887.

[8] Y. Watanabe et al., Proceedings of the International Conference on $\mathrm{Nu}-$ clear Data for Science and Technology, Jeju Island, Korea, April 26-30, 2010, J. Korean Phys. Soc. 59 (2011) 1040.

[9] C. Paradela et al. (the n_TOF Collaboration), Phys. Rev. C 82 (2010) 034601. 
[10] D. Tarrío et al. (the n_TOF Collaboration), Phys. Rev. C 83 (2011) 044620 .

[11] M. I. Baznat, K. K. Gudima and S. G. Mashnik, Proceedings of the AccApp03 Meeting, San Diego, California, June 1-5 2003, p. 976; arXiv: nucl-th/0307014.

[12] S. G. Mashnik, K. K. Gudima, R. E. Prael and A. J. Sierk, Proceedings of the Workshop on Nuclear Data for the Transmutation of Nuclear Waste, LANL report LA-UR-04-1873, Los Alamos, 2004; arXiv: nuclth/0404018.

[13] S. G. Mashnik, A. J. Sierk and R. E. Prael, Proceedings of the International Conference on Nuclear Data for Science and Technology, New York, March 4-8 2013, Nucl. Data Sheets 118 (2014) 320.

[14] A. Boudard, J. Cugnon, J.-C.David, S. Leray and D. Mancusi, Phys. Rev. C 87 (2013) 014606.

[15] J. Cugnon, C. Volant and S. Vuillier, Nucl. Phys. A 620 (1997) 475.

[16] http://www-nds.iaea.org/spallations

[17] S. Leray et al., Proceedings of the International Conference on Nuclear Data for Science and Technology, Jeju Island, Korea, April 26-30, 2010, J. Korean Phys. Soc. 59 (2011) 791.

[18] D. Mancusi, in preparation.

[19] R. J. Charity, Proceedings of the Joint ICTP-IAEA Advanced Workshop on Model Codes for Spallation Reactions, ICTP Trieste, Italy, 4-8 February 2008, D. Filges et al., Eds., IAEA report INDC(NDS)-530, Vienna, p. 139 (2008).

[20] D. Mancusi, R. J. Charity and J. Cugnon, Phys. Rev. C 82 (2010) 044610 .

[21] A. Kelić, M. V. Ricciardi and K. H. Schmidt, Proceedings of the Joint ICTP-IAEA Advanced Workshop on Model Codes for Spallation Reactions, ICTP Trieste, Italy, 4-8 February 2008, D. Filges et al., Eds., IAEA report INDC(NDS)-530, Vienna, p. 181 (2008). 
[22] K. K. Gudima, S. G. Mashnik and V. D. Toneev, Nucl. Phys. A 401 (1983) 329.

[23] S. G. Mashnik, K. K. Gudima, R. E. Prael, A. J. Sierk, M. I. Baznat and N. V. Mokhov, LANL report LA-UR-08-2931, Los Alamos, 2008; arXiv: 0805.0751 .

[24] A. V. Ignatyuk, G. N. Smirenkin and A. S. Tishin, Sov. J. Nucl. Phys. 21 (1975) 255.

[25] R. J. Charity, Phys. Rev. C 82 (2010) 014610.

[26] L. G. Moretto, Nucl. Phys. A 247 (1975) 221.

[27] L. G. Moretto and G. J. Wozniak, Prog. Part. Nucl. Phys. 21 (1988) 401.

[28] A. J. Sierk, Phys. Rev. C 33 (1986) 2039.

[29] P. Möller et al., Atomic Data Nucl. Data Tables 59 (1995) 185.

[30] A. R. Junghans, M. De Jong, H. G. Clerc, A. V. Ignatyuk, G. A. Kudyaev and K. H. Schmidt, Nucl. Phys. A 629 (1998) 635.

[31] V. E. Bunakov, L. V. Krasnov and A. V. Fomichev, Eur. Phys. J. A 8 (2000) 447.

[32] S. G. Mashnik, User Manual for the Code CEM95, IAEA report 1247/01, Vienna, 1995.

[33] A. V. Prokofiev, S. G. Mashnik and A. J. Sierk, Nucl. Sci. Eng. 131 (1999) 78.

[34] A. N. Smirnov et al., Proceedings of the International Conference on Nuclear Data for Science and Technology, Tsukuba, Japan, October 712, 2001, J. Nucl. Sci. Technol. Suppl. 2 (2002) 238.

[35] S. Lo Meo, D. Mancusi, C. Massimi, G. Vannini and A. Ventura, Proceedings of the $20^{\text {th }}$ International School on Nuclear Physics, Neutron Physics and Applications, Varna, Bulgaria, September 17-21, 2013; J. Phys. : Conf. Ser. 533 (2014) 012024. 
[36] H. M. Steiner and J. A. Jungerman, Phys. Rev. 101 (1956) 807.

[37] R. Brandt et al., Revue de Physique Appliquée 7 (1972) 243.

[38] V. A. Konshin, E. S. Matusevich and V. I. Regushevskii, Sov. J. Nucl. Phys. 2 (1966) 489.

[39] V. I. Yurevich, V. A. Nikolaev, R. M. Yakovlev and A. N. Sosnin, Phys. At. Nucl. 65 (2002) 1383.

[40] P. W. Lisowski et al., Proceedings of the Specialist's Meeting on Neutron Cross Section Standards for the Energy Region above $20 \mathrm{MeV}$, Uppsala, Sweden, 1991, NEA report NEANDC-305/U, p. 177.

[41] R. Nolte et al., Nucl. Sci. Eng. 156 (2007) 197.

[42] A. D. Carlson, S. Chiba, F. J. Hambsch, N. Olsson and A. N. Smirnov, Proceedings of the International Conference on Nuclear Data for Science and Technology, Trieste, Italy, May 19-24, 1997, Part II, p. 1223 (1997).

[43] J. Rapaport et al., LANL report LA-11078-MS, Los Alamos, p. 1 (1987).

[44] K.-H. Schmidt et al., Phys. Rev. C 87 (2013) 034601.

[45] B. A. Bochagov et al., Sov. J. Nucl. Phys. 28 (1978) 291.

[46] O. E. Shigaev et al., Khlopin Radiev. Inst. report RI-17 (1973).

[47] V. S. Bychenkov et al., Sov. J. Nucl. Phys. 17 (1973) 496.

[48] O. A. Shcherbakov et al., Proceedings of the International Conference on Nuclear Data for Science and Technology, Tsukuba, Japan, October 7-12, 2001, J. Nucl. Sci. Technol. Suppl. 2 (2002) 230.

[49] A. D. Carlson, S. Chiba, F. J. Hambsch, N. Olsson and A. N. Smirnov, IAEA report INDC(NDS)-368, Vienna, p. 23 (1997).

[50] A. V. Prokofiev et al., Phys. Rev. C 53 (1996) 2911.

[51] V. I. Goldanskii, E. Z. Tarumov and V. S. Penkina, Dokl. Akad. Nauk 101 (1955) 1027. 
[52] T. Fukahori et al., Proceedings of the International Conference on $\mathrm{Nu}-$ clear Data for Science and Technology, Tsukuba, Japan, October 7-12, 2001, J. Nucl. Sci. Technol. Suppl. 2 (2002) 25.

[53] J. Hudis and S. Katcoff, Phys. Rev. C 13 (1976) 1961.

[54] M. Debeauvais, J. Tripler ans S. Jokic, Proceedings of the $9^{\text {th }}$ International Conference on Solid State Nuclear Track Detectors, Neuherberg, Germany, 1976, vol. 2, p. 1179.

[55] G. Remy, J. Ralarosy, R. Stein, M. Debeauvais and J. Tripier, Nucl. Phys. A 163 (1971) 583.

[56] L. Kowalski and C. Stephan, J. de Physique 24 (1963) 901.

[57] V. P. Shamov, Dokl. Akad. Nauk 103 (1955) 593.

[58] F. Saint-Laurent, M. Conjeaud, R. Dayras, S. Harar, H. Oeschler and C. Volant, Nucl. Phys. A 422 (1984) 307.

[59] A. P. Vinogradov et al., Proceed. of the International Conference on Peaceful Uses of Atomic Energy, Moscow, 1955, p. 97.

[60] H. U. Wenger et al., Nucl. Phys. A 764 (2006) 1.

[61] P. W. Lisowski et al., Proceed. of the International Conference on Nuclear Data for Science and Technology, Jülich (Germany), 1991, p. 732.

[62] D. Karadimos et al. (the n_TOF Collaboration), Phys. Rev. C 89 (2014) 044606.

[63] C. Paradela et al. (the $\mathrm{n}_{-}$TOF Collaboration), Proceedings of the International Conference on Advances in Nuclear Analysis and Simulations, Vancouver, Canada, 2006, B076.

[64] P. W. Lisowski et al., Proceedings of the International Conference on Nuclear Data for Science and Technology, Mito, Japan, 1988, p. 97.

[65] G. S. Karapetyan, A. R. Balabekyan, N. A. Demekhina and J. Adam, Phys. At. Nucl. 72 (2009) 911.

[66] F. Tovesson and T. S. Hill, Phys. Rev. C 75 (2007) 034610. 
[67] F. Tovesson and T. S. Hill, Nucl. Sci. Eng. 165 (2010) 224.

[68] L. A. Vaishnene et al., Bull. Russ. Acad. Sci. - Physics 74 (2010) 496.

[69] G. N. Flerov, V. P. Perelygin and O. Otgonsurén, Sov. At. En. 33 (1972) 1144 .

[70] T. Enqvist et al., Nucl. Phys. A 686 (2001) 481.

[71] A. N. Smirnov et al., Phys. Rev. C 70 (2004) 054603.

[72] I. V. Rhyzov et al., Nucl. Instr. Meth. Phys. Res. A 562 (2006) 439.

[73] H. G. De Carvalho, G. Potenza, R. Rinzivillo, E. Sassi and G. Vanderhaeghe, Nuovo Cimento 25 (1962) 880.

[74] A. V. Fomichev, V. N. Dushin, S. M. Soloviev, A. A. Fomichev and S. Mashnik, Khlopin Radiev. Inst. report RI-262 (2004).

[75] A. B. Laptev et al., Proceedings of the International Conference on Fission Properties of Neutron Rich Nuclei, Sanibel Island, 2007, p. 462.

[76] O. T. Grudzevich and S. G. Yavshits, Phys. At. Nucl. 76 (2013) 263. 